DOI 10.4171/JEMS/511

Joan Bagaria · Carles Casacuberta · A. R. D. Mathias · Jiří Rosický

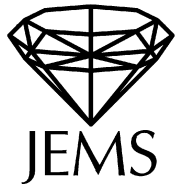

\title{
Definable orthogonality classes in accessible categories are small
}

Received February 17, 2011 and in revised form December 2, 2012

\begin{abstract}
We lower substantially the strength of the assumptions needed for the validity of certain results in category theory and homotopy theory which were known to follow from Vopěnka's principle. We prove that the necessary large-cardinal hypotheses depend on the complexity of the formulas defining the given classes, in the sense of the Lévy hierarchy. For example, the statement that, for a class $\mathcal{S}$ of morphisms in a locally presentable category $\mathcal{C}$ of structures, the orthogonal class of objects is a small-orthogonality class (hence reflective) can be proved in ZFC if $\mathcal{S}$ is $\boldsymbol{\Sigma}_{1}$, while it follows from the existence of a proper class of supercompact cardinals if $\mathcal{S}$ is $\boldsymbol{\Sigma}_{2}$, and from the existence of a proper class of what we call $C(n)$-extendible cardinals if $\mathcal{S}$ is $\boldsymbol{\Sigma}_{n+2}$ for $n \geq 1$. These cardinals form a new hierarchy, and we show that Vopěnka's principle is equivalent to the existence of $C(n)$-extendible cardinals for all $n$. As a consequence of our approach, we prove that the existence of cohomological localizations of simplicial sets, a long-standing open problem in algebraic topology, is implied by the existence of arbitrarily large supercompact cardinals. This follows from the fact that $E^{*}$-equivalence classes are $\boldsymbol{\Sigma}_{2}$, where $E$ denotes a spectrum treated as a parameter. In contrast with this fact, $E_{*}$-equivalence classes are $\Sigma_{1}$, from which it follows (as is well known) that the existence of homological localizations is provable in ZFC.
\end{abstract}

Keywords. Supercompact cardinal, extendible cardinal, Lévy hierarchy, accessible category, reflective subcategory, cohomological localization

\section{Introduction}

The answers to certain questions in category theory turn out to depend on set theory. A typical example is whether every full limit-closed subcategory of a complete category

J. Bagaria: ICREA (Institució Catalana de Recerca i Estudis Avançats) and Departament de Lògica, Història i Filosofia de la Ciència, Universitat de Barcelona, Montalegre 6, 08001 Barcelona, Spain; e-mail: joan.bagaria@icrea.cat, bagaria@ub.edu

C. Casacuberta: Institut de Matemàtica, Universitat de Barcelona, Gran Via de les Corts Catalanes 585, 08007 Barcelona, Spain; e-mail: carles.casacuberta@ub.edu

A. R. D. Mathias: ERMIT, Université de la Réunion, UFR Sciences et Technologies, Laboratoire d'Informatique et de Mathématiques, 2 rue Joseph Wetzel, Bâtiment 2, F-97490 Sainte Clotilde, France outre-mer; e-mail: ardm@dpmms.cam.ac.uk, ardm@email.mathematik.uni-freiburg.de

J. Rosický: Department of Mathematics and Statistics, Masaryk University, Kotlárská 2, 60000 Brno, Czech Republic; e-mail: rosicky@math.muni.cz

Mathematics Subject Classification (2010): Primary 03E55, 18A40, 55P60; Secondary 03C55, $18 \mathrm{C} 35$ 
$\mathcal{C}$ is reflective. On the one hand, there are counterexamples involving the category of topological spaces and continuous functions [45]. On the other hand, as explained in [2], an affirmative answer to this question for locally presentable categories is implied by a large-cardinal axiom called Vopěnka's principle (stating that, for every proper class of structures of the same type, there exists a nontrivial elementary embedding between two of them).

Large cardinals were used in a similar way in [17] to show that the existence of cohomological localizations, a famous unsolved problem, follows from Vopěnka's principle. Other relevant consequences of Vopěnka's principle in algebraic topology were found in [15], [16], [19], [43]. However, the precise consistency strength of many implications of this axiom in category theory or homotopy theory is not known, and in some cases the question of whether such statements are provable in ZFC remains unanswered. A relevant step in this direction was made in [42].

In another direction, it was pointed out in [9] that certain results about accessible categories that follow from Vopěnka's principle are still true under much weaker largecardinal assumptions. This claim is based on the following finding, which is the subject of the present article: the assumptions needed to infer reflectivity or smallness of orthogonality classes in accessible categories may depend on the complexity of the formulas in the language of set theory defining these classes. Here "complexity" is meant in the sense of the Lévy hierarchy [31, Ch. 13]. Recall that $\Sigma_{n}$ formulas and $\Pi_{n}$ formulas are defined inductively as follows: $\Pi_{0}$ formulas are the same as $\Sigma_{0}$ formulas, namely formulas in which all quantifiers are bounded; $\Sigma_{n+1}$ formulas are of the form $\exists x \varphi$ where $\varphi$ is $\Pi_{n}$, and $\Pi_{n+1}$ formulas are of the form $\forall x \varphi$ where $\varphi$ is $\Sigma_{n}$.

For example, as we prove in this article, if $\mathcal{S}$ is a full limit-closed subcategory of a locally presentable category $\mathcal{C}$ of structures, and $\mathcal{S}$ can be defined with a $\Sigma_{2}$ formula (possibly with parameters), then the existence of a proper class of supercompact cardinals suffices to ensure reflectivity of $\mathcal{S}$. Moreover, remarkably, if $\mathcal{S}$ can be defined with a $\Sigma_{1}$ formula, then the reflectivity of $\mathcal{S}$ is provable in ZFC.

In the case of a more complex definition of $\mathcal{S}$, its reflectivity follows from the existence of a proper class of what we call $C(n)$-extendible cardinals, for some $n$. These cardinals form a natural hierarchy ranging from extendible cardinals [31, 20.22] when $n=1$ to Vopěnka's principle. Indeed, as stated in Corollary 6.9 below, Vopěnka's principle is equivalent to the claim that there exists a $C(n)$-extendible cardinal for every $n<\omega$. We denote by $C(n)$ the proper class of cardinals $\alpha$ such that $V_{\alpha}$ is a $\Sigma_{n}$-elementary submodel of the set-theoretic universe $V$, and say that a cardinal $\kappa$ is $C(n)$-extendible if $\kappa \in C(n)$ and for all $\lambda>\kappa$ in $C(n)$ there is an elementary embedding $j: V_{\lambda} \rightarrow V_{\mu}$ for some $\mu \in C(n)$ with critical point $\kappa$, such that $j(\kappa) \in C(n)$ and $j(\kappa)>\lambda$.

By way of this approach, we prove that the existence of cohomological localizations of simplicial sets follows from the existence of a proper class of supercompact cardinals. This result uses the fact, proved in Theorem 9.3 below, that for every (BousfieldFriedlander) spectrum $E$ the class of $E^{*}$-acyclic simplicial sets (where $E^{*}$ denotes the reduced cohomology theory represented by $E$ ) can be defined by means of a $\Sigma_{2}$ formula with $E$ as a parameter. However, the class of $E_{*}$-acyclic simplicial sets (where $E_{*}$ now denotes homology) can be defined with a $\Sigma_{1}$ formula. This is consistent with the fact 
that the existence of homological localizations can be proved in ZFC, as done indeed by Bousfield [11]; see also [5].

The reason why classes of homology acyclics have lower complexity than classes of cohomology acyclics is that, for a fibrant simplicial set $Y$ with basepoint, the statement "all pointed maps $f: \mathbb{S}^{n} \rightarrow Y$ are nullhomotopic", where $\mathbb{S}^{n}$ is the simplicial $n$-sphere, is absolute between transitive models of ZFC, since a simplicial map $\mathbb{S}^{n} \rightarrow Y$ is determined by a single $n$-simplex of $Y$ satisfying certain conditions expressible in terms of $Y$ with bounded quantifiers (cf. [40,3.6]). However, if $X$ and $Y$ are simplicial sets with basepoints $x_{0}$ and $y_{0}$, then the statement "all pointed maps $f: X \rightarrow Y$ are nullhomotopic" involves unbounded quantifiers, since it is formalized, for example, by stating that

$$
\forall f\left(f \text { is a map from } X \text { to } Y \rightarrow \exists h \text { ( } h \text { is a homotopy from } f \text { to } y_{0}\right) \text { ). }
$$

Therefore, for a spectrum $E$, there might exist $E^{*}$-acyclic simplicial sets in a transitive model of ZFC containing $E$ that fail to be $E^{*}$-acyclic in some larger model, while the class of $E_{*}$-acyclic simplicial sets is absolute. See Section 9 for a detailed discussion of these facts.

Another consequence of this article is that the main theorem of [9] can now be proved for reflections, not necessarily epireflections. Thus, if there are arbitrarily large supercompact cardinals, then every reflection $L$ on an accessible category of structures is an $\mathcal{F}$-reflection for some set $\mathcal{F}$ of morphisms, provided that the class of $L$-equivalences is $\boldsymbol{\Sigma}_{2}$ (see Corollary 8.5 below). Boldface types $\boldsymbol{\Sigma}_{n}$ or $\Pi_{n}$ are used to denote the fact that the corresponding formulas may contain parameters.

We also prove that the Freyd-Kelly orthogonal subcategory problem [25], asking if $\mathcal{S}^{\perp}$ is reflective for a class of morphisms $\mathcal{S}$ in a suitable category, has an affirmative answer in ZFC for $\boldsymbol{\Sigma}_{1}$ classes in locally presentable categories of structures. It is also true for $\boldsymbol{\Sigma}_{2}$ classes if a proper class of supercompact cardinals is assumed to exist, and for $\boldsymbol{\Sigma}_{n+2}$ classes if there is a proper class of $C(n)$-extendible cardinals for $n \geq 1$. We say that $\mathcal{S}$ is definable with sufficiently low complexity to encompass all these cases in a single phrase.

Essentially the same arguments hold in the homotopy category of simplicial sets, hence yielding a simpler and more accurate answer than in [17] (where Vopěnka's principle was used) to Farjoun's question in [20] of whether every homotopy reflection on simplicial sets is an $f$-localization for some map $f$. Localizations with respect to sets of maps were constructed in [12], [21], [28], and the extension to proper classes of maps was carried out in [17] using Vopěnka's principle. Here we prove that localizations with respect to proper classes of maps exist whenever the given classes are definable with sufficiently low complexity.

We warn the reader that in this article, as well as in [9], complexity of classes of objects or morphisms in an accessible category $\mathcal{C}$ is meant under the assumption that $\mathcal{C}$ is accessibly embedded into a category of structures. This happens canonically with the category of simplicial sets and with the category of Bousfield-Friedlander spectra, or, more generally, with categories of models of basic theories in any language. Terminology and background can be found in [2,5.B], where it is proved that every accessible category is equivalent to one which is accessibly embedded into a category of structures. 


\section{Categories of structures}

Most of the results in this article refer to categories of structures (possibly many-sorted, in a language of any cardinality). For the convenience of the reader, we start by recalling terminology and background about structures and models in this section. Additional details can be found, among many other sources, in [2, Ch. 5] and [31, Ch. 12].

For a regular cardinal $\lambda$, a $\lambda$-ary $S$-sorted signature $\Sigma$ consists of a set $S$ of sorts, a set $\Sigma_{\text {op }}$ of operation symbols, another set $\Sigma_{\text {rel }}$ of relation symbols, and an arity function that assigns to each operation symbol an ordinal $\alpha<\lambda$, a sequence $\left\langle s_{i}: i \in \alpha\right\rangle$ of input sorts and an output sort $s \in S$, and to each relation symbol an ordinal $\beta<\lambda$ and a sequence of sorts $\left\langle s_{j}: j \in \beta\right\rangle$. An operation symbol with $\alpha=\emptyset$ is called a constant symbol. A signature $\Sigma$ is called operational if $\Sigma_{\mathrm{rel}}=\emptyset$ and relational if $\Sigma_{\mathrm{op}}=\emptyset$.

Given an $S$-sorted signature $\Sigma$, a $\Sigma$-structure is a triple

$$
X=\left\langle\left\{X_{s}: s \in S\right\},\left\{\sigma_{X}: \sigma \in \Sigma_{\mathrm{op}}\right\},\left\{\rho_{X}: \rho \in \Sigma_{\mathrm{rel}}\right\}\right\rangle
$$

consisting of an underlying $S$-sorted set or universe, denoted by $\left\{X_{s}: s \in S\right\}$ or $\left(X_{S}\right)_{s \in S}$, together with a function

$$
\sigma_{X}: \prod_{i \in \alpha} X_{s_{i}} \rightarrow X_{s}
$$

for each operation symbol $\sigma \in \Sigma_{\mathrm{op}}$ of arity $\left\langle s_{i}: i \in \alpha\right\rangle \rightarrow s$ (including a distinguished element of $X_{s}$ for each constant symbol of sort $s$ ), and a set

$$
\rho_{X} \subseteq \prod_{j \in \beta} X_{s_{j}}
$$

for each relation symbol $\rho \in \Sigma_{\text {rel }}$ of arity $\left\langle s_{j}: j \in \beta\right\rangle$.

A homomorphism $f: X \rightarrow Y$ between two $\Sigma$-structures is an $S$-sorted function $\left(f_{S}: X_{S} \rightarrow Y_{S}\right)_{S \in S}$ preserving operations and relations. For each signature $\Sigma$, the category of $\Sigma$-structures and their homomorphisms will be denoted by $\operatorname{Str} \Sigma$.

Given a $\lambda$-ary $S$-sorted signature $\Sigma$, the language $\mathcal{L}_{\lambda}(\Sigma)$ consists of sets of variables, terms, and formulas, which are defined as follows. There is a family $W=\left\{W_{s}: s \in S\right\}$ of sets of cardinality $\lambda$, the elements of $W_{s}$ being variables of sort $s$. One defines terms by declaring that each variable is a term and, for each operation symbol $\sigma \in \Sigma_{\text {op }}$ of arity $\left\langle s_{i}: i \in \alpha\right\rangle \rightarrow s$ and each collection of terms $\tau_{i}$ of sort $s_{i}$, the expression $\sigma\left(\tau_{i}\right)_{i \in \alpha}$ is a term of sort $s$. Atomic formulas are expressions of the form $\tau_{1}=\tau_{2}$ and $\rho\left(\tau_{j}\right)_{j \in \beta}$, where $\rho \in \Sigma_{\text {rel }}$ is a relation symbol of arity $\left\langle s_{j}: j \in \beta\right\rangle$ and each $\tau_{j}$ is a term of sort $s_{j}$ with $j \in \beta$. Formulas are built in finitely many steps from the atomic formulas by means of logical connectives and quantifiers. Thus, if $\left\{\varphi_{i}: i \in I\right\}$ are formulas and $|I|<\lambda$, then so are the conjunction $\bigwedge_{i \in I} \varphi_{i}$ and the disjunction $\bigvee_{i \in I} \varphi_{i}$. Quantification is allowed over sets of variables of cardinality smaller than $\lambda$; that is, $\left(\forall\left(x_{i}\right)_{i \in I}\right) \varphi$ and $\left(\exists\left(x_{i}\right)_{i \in I}\right) \varphi$ are formulas if $\varphi$ is a formula and $|I|<\lambda$.

Variables that appear unquantified in a formula are called free. If a formula is denoted by $\varphi\left(x_{i}\right)_{i \in I}$, it is meant that each $x_{i}$ is a free variable. 
Each language $\mathcal{L}_{\lambda}(\Sigma)$ determines a satisfaction relation between $\Sigma$-structures and formulas with an assignment for their free variables. If $\varphi\left(x_{i}\right)_{i \in I}$ is a formula where each $x_{i}$ is a free variable of sort $s_{i}$ and $X$ is a $\Sigma$-structure, a variable assignment, denoted by $x_{i} \mapsto a_{i}$, is a function $a: I \rightarrow \bigcup_{s \in S} X_{S}$ such that $a(i) \in X_{s_{i}}$ for all $i$. Satisfaction of a formula $\varphi$ in a $\Sigma$-structure $X$ is defined inductively, starting with the atomic formulas and quantifying over subsets of $\bigcup_{s \in S} X_{s}$ of cardinality smaller than $\lambda$ (see [2, §5.26] for details). We write $X \models \varphi\left(a_{i}\right)_{i \in I}$ if $\varphi$ is satisfied in $X$ under an assignment $x_{i} \mapsto a_{i}$ for all its free variables $x_{i}$.

A formula without free variables is called a sentence. A set of sentences is called a theory. A model of a theory $T$ in a language $\mathcal{L}_{\lambda}(\Sigma)$ is a $\Sigma$-structure satisfying all sentences of $T$. For each theory $T$, we denote by $\operatorname{Mod} T$ the full subcategory of $\operatorname{Str} \Sigma$ consisting of all models of $T$.

A language $\mathcal{L}_{\lambda}(\Sigma)$ is called finitary if $\lambda=\omega$ (the least infinite cardinal); otherwise it is infinitary. An especially important finitary language is the language of set theory. This is the first-order finitary language corresponding to the signature with one sort, namely "sets", and one binary relation symbol ("membership"). Hence the atomic formulas are $x=y$ and $x \in y$, where $x$ and $y$ are sets.

Define, recursively on the class of ordinals, $V_{0}=\emptyset, V_{\alpha+1}=\mathcal{P}\left(V_{\alpha}\right)$ for all $\alpha$, where $\mathcal{P}$ denotes the power-set operation, and $V_{\lambda}=\bigcup_{\alpha<\lambda} V_{\alpha}$ if $\lambda$ is a limit ordinal. Then every set is an element of some $V_{\alpha}$ (see [30, Lemma 9.3] or [31, Lemma 6.3]). The rank of a set $X$ is defined as the least ordinal $\alpha$ such that $X \in V_{\alpha+1}$. Hence $V_{\alpha}$ is the set of all sets whose rank is less than $\alpha$. The universe $V$ of all sets is the union of $V_{\alpha}$ for all ordinals $\alpha$.

Everything in this article is formulated in ZFC (Zermelo-Fraenkel set theory with the axiom of choice). Thus, a class consists of all sets for which a certain formula of the language of set theory is satisfied, possibly with parameters. More precisely, a class $\mathcal{C}$ is defined by a formula $\varphi\left(x, y_{1}, \ldots, y_{n}\right)$ with parameters $p_{1}, \ldots, p_{n}$ if

$$
\mathcal{C}=\left\{x: \varphi\left(x, p_{1}, \ldots, p_{n}\right)\right\}
$$

where satisfaction, if unspecified, is meant in the universe $V$. The sets $p_{1}, \ldots, p_{n}$ are fixed values of $y_{1}, \ldots, y_{n}$ under every variable assignment. To simplify the notation, we often replace $p_{1}, \ldots, p_{n}$ by a single parameter $p=\left\{p_{1}, \ldots, p_{n}\right\}$. A class which is not a set is called a proper class. Each set $A$ is definable with $A$ itself as a parameter by $A=\{x: x \in A\}$.

In this article, a model of $Z F C$ will be a pair $\langle M, \in\rangle$ where $M$ is a set or a proper class and $\in$ is the restriction of the membership relation to $M$, in which the formalized ZFC axioms are satisfied. Thus, if we neglect the fact that $M$ can be a proper class, we may view $\langle M, \in\rangle$ as a $\Sigma$-structure where $\Sigma$ is the relational signature of the language of set theory, and in fact a model of the theory consisting of the formalized ZFC axioms. In particular, $\langle V, \in\rangle$ itself is such a model.

A class $M$ is transitive if every element of an element of $M$ is an element of $M$. We shall always assume that models of ZFC are transitive, but not necessarily inner (a model is called inner if it is transitive and contains all the ordinals). 


\section{The Lévy hierarchy}

In this section we specialize to the language of set theory. Given two classes $M \subseteq N$, we say that a formula $\varphi\left(x_{1}, \ldots, x_{k}\right)$ is absolute between $M$ and $N$ if, for all $a_{1}, \ldots, \bar{a}_{k}$ in $M$,

$$
N \models \varphi\left(a_{1}, \ldots, a_{k}\right) \quad \text { if and only if } \quad M \models \varphi\left(a_{1}, \ldots, a_{k}\right) .
$$

We say that a formula $\varphi\left(x_{1}, \ldots, x_{k}\right)$ is upward absolute for transitive models of some theory $T$ if, given any two such models $M \subseteq N$ and given $a_{1}, \ldots, a_{k} \in M$ for which $\varphi\left(a_{1}, \ldots, a_{k}\right)$ is true in $M, \varphi\left(a_{1}, \ldots, a_{k}\right)$ is also true in $N$. And we say that $\varphi$ is downward absolute if, in the same situation, if $\varphi\left(a_{1}, \ldots, a_{k}\right)$ holds in $N$ then it holds in $M$. A formula is absolute if it is both upward and downward absolute. If $T$ is unspecified, then it should be understood that $T$ is by default the set of all formalized ZFC axioms. If it is meant, on the contrary, that $T=\emptyset$, then we speak of absoluteness between transitive classes.

A class $\mathcal{C}$ is upward absolute between transitive classes $M \subseteq N$ if it is definable, possibly with a set $p$ of parameters, by a formula that is upward absolute between $M$ and N. Downward absolute classes are defined analogously, and we say that $\mathcal{C}$ is absolute between $M$ and $N$ if it is upward absolute and downward absolute, hence allowing the possibility that $\mathcal{C}=\{x: \varphi(x, p)\}=\{x: \psi(x, p)\}$ where $\varphi$ is upward absolute and $\psi$ is downward absolute. In this situation, $N \models x \in \mathcal{C}$ if and only if $M \models x \in \mathcal{C}$, assuming that $p \in M$.

The following terminology is due to Lévy (see [31, Ch. 13]). A formula of the language of set theory is said to be $\Sigma_{0}$ if all its quantifiers are bounded, that is, of the form $\exists x \in a$ or $\forall x \in a$. Then $\Sigma_{n}$ formulas and $\Pi_{n}$ formulas are defined inductively as follows: $\Pi_{0}$ formulas are the same as $\Sigma_{0}$ formulas; $\Sigma_{n+1}$ formulas are of the form $\left(\exists x_{1} \ldots x_{k}\right) \varphi$, where $\varphi$ is $\Pi_{n}$; and $\Pi_{n+1}$ formulas are of the form $\left(\forall x_{1} \ldots x_{k}\right) \varphi$, where $\varphi$ is $\Sigma_{n}$. We say that a formula is $\Sigma_{n} \wedge \Pi_{n}$ if it is a conjunction of a $\Sigma_{n}$ formula and a $\Pi_{n}$ formula.

Classes can be defined by distinct formulas and, more generally, properties and mathematical statements can be formalized in the language of set theory in many different ways. We say that a class $\mathcal{C}$ is $\boldsymbol{\Sigma}_{n}$-definable (or, briefly, that $\mathcal{C}$ is $\boldsymbol{\Sigma}_{n}$ ) if there is a $\Sigma_{n}$ formula $\varphi(x, y)$ such that $\mathcal{C}=\{x: \varphi(x, p)\}$ for a set $p$ of parameters. Similarly, a class is $\Pi_{n}$ if it can be defined by some $\Pi_{n}$ formula with parameters. A class is called $\boldsymbol{\Delta}_{n}$ if it is both $\boldsymbol{\Sigma}_{n}$ and $\boldsymbol{\Pi}_{n}$. For notational convenience, if no parameters are involved, then we write that a class $\mathcal{C}$ is $\Sigma_{n}, \Pi_{n}$ or $\Delta_{n}$, using lightface types.

The same terminology is used with statements or informal expressions; for example, " $\lambda$ is a cardinal" is a $\Pi_{1}$ statement [31, Lemma 13.13], while " $f$ is a function", " $\alpha$ is an ordinal" or " $\omega$ is the least nonzero limit ordinal" are $\Delta_{0}$ statements [31, Lemma 12.10].

If a class $\mathcal{C}$ is $\Sigma_{1}$ with a set $p$ of parameters, then it is upward absolute for transitive classes containing $p$. In fact, given a $\Sigma_{1}$ formula $\exists x \varphi(x, y)$ where $\varphi$ is $\Sigma_{0}$ and given a set $p$ of parameters, suppose that $M \subseteq N$ are transitive classes with $p \in M$. Then, if $M \models \exists x \varphi(x, p)$, we may infer that $N \models \exists x \varphi(x, p)$ as well, since if $a \in M$ witnesses that $\varphi(a, p)$ holds in $M$, then $a \in N$ and $\varphi(a, p)$ also holds in $N$, since $\varphi$ is absolute.

Conversely, if a class $\mathcal{C}$ is upward absolute for transitive models of some finite fragment $\mathrm{ZFC}^{*}$ of $\mathrm{ZFC}$, then it is $\boldsymbol{\Sigma}_{1}$. To prove this claim, suppose that $\mathcal{C}$ is defined by a 
formula $\varphi(x, y)$ that is upward absolute for transitive models of $\mathrm{ZFC}^{*}$ with a set $p$ of parameters. Then $\mathcal{C}$ is also defined by the following $\Sigma_{1}$ formula:

$$
\exists M\left[M \text { is transitive } \wedge\{x, p\} \subset M \wedge M \models\left(\varphi(x, p) \wedge\left(\bigwedge \mathrm{ZFC}^{*}\right)\right)\right] .
$$

Indeed, if $a \in \mathcal{C}$ then $\varphi(a, p)$ holds in $V$, and it follows from the Reflection Principle [31, Theorem 12.14] that there is an ordinal $\alpha$ with $\{a, p\} \in V_{\alpha}$ such that $V_{\alpha} \models \varphi(a, p)$ and all the sentences in the finite set ZFC* are satisfied in $V_{\alpha}$, so $V_{\alpha}$ witnesses (2.1). And, if a set $M$ witnesses (2.1) for some variable assignment $x \mapsto a$, then, since $\varphi(x, y)$ is upward absolute for transitive models of $\mathrm{ZFC}^{*}$, we infer that $\varphi(a, p)$ holds in $V$, that is, $a \in \mathcal{C}$.

Similarly, if a class $\mathcal{C}$ is defined by a $\Pi_{1}$ formula with parameters, then it is downward absolute for transitive classes containing the parameters, and, if $\mathcal{C}$ is downward absolute for transitive models of some finite fragment of ZFC, then it is $\Pi_{1}$, analogously to (2.1). We conclude that $\boldsymbol{\Delta}_{1}$ classes are absolute for transitive classes containing the parameters.

The following are examples of nonabsoluteness which will be relevant in this article.

Example 2.1. The class of topological spaces is $\Pi_{1}$, since the union of every collection of open sets must be open. Thus, a topology on a set $X$ in some model of ZFC may fail to be a topology on $X$ in a larger model. However, the class of simplicial sets is $\Delta_{0}$ (see Section 9).

Example 2.2. Let $\mathcal{C}$ be the class of all abelian groups of the form $\mathbb{Z}^{\kappa}$, where $\kappa$ is a cardinal. Then $A \in \mathcal{C}$ if and only if

$$
\exists x(x \text { is a cardinal } \wedge \forall y(y \in A \leftrightarrow y \text { is a function from } x \text { to } \mathbb{Z})),
$$

which is a $\Sigma_{2}$ formula, since the expression written within the outer parentheses is $\Pi_{1}$. In every model of ZFC with measurable cardinals, the following sentence is true:

$\exists \kappa \exists f$ ( $\kappa$ is an infinite cardinal $\wedge f$ is a group homomorphism from $\mathbb{Z}^{\kappa}$ to $\mathbb{Z}$

$$
\left.\wedge f\left(\mathbb{Z}^{<\kappa}\right)=0 \wedge f \neq 0\right),
$$

while if this holds then the smallest $\kappa$ with this property is measurable, according to [22]; see [23] for further details. Therefore, this sentence is false in a model of ZFC without measurable cardinals while it is true in a model of ZFC with measurable cardinals.

Example 2.3. For a cardinal $\lambda$ and a set $X$, we denote by $\mathcal{P}_{\lambda}(X)$ the set of all subsets of $X$ whose cardinality is smaller than $\lambda$. Note first that, although the statement " $A$ is a subset of $B$ " is $\Delta_{0}$, the statement " $A$ is the set of all subsets of $B$ " is formalized with the following $\Pi_{1}$ formula:

$$
\forall a \in A(a \subseteq B) \wedge \forall x(x \subseteq B \rightarrow x \in A) .
$$

This statement cannot be formalized with any upward absolute formula, since, if we pick a countable transitive model $M$ of ZFC and $A$ is the set of all subsets of the natural numbers $\mathbb{N}$ in $M$, then $A$ cannot be the set of all subsets of $\mathbb{N}$ in the universe $V$, since $A$ is countable. 
The assertion " $x$ is finite" is $\Delta_{1}$, since it is equivalent to the statement that there exists a bijection between $x$ and a finite ordinal (which is $\Sigma_{1}$ ) and it is also equivalent to the statement that every injective function from $x$ to itself is surjective (which is $\Pi_{1}$ ). Note also that, if a set $x$ is finite and each of its elements belongs to a model $M$ of ZFC, then we may infer that $x \in M$ using the pairing and union axioms. From this fact it follows that the statement $A=\mathcal{P}_{\omega}(B)$-that is, " $A$ is the set of all finite subsets of $B$ "-is absolute for transitive models of a suitable finite fragment of ZFC, hence $\Delta_{1}$. Nevertheless, if $M$ and $N$ are just transitive classes with $M \subset N$ and $B \in M$, it can happen that the claim " $\mathcal{P}_{\omega}(B)$ exists" is true in $N$ but not in $M$, as discussed in [39, Sections 5 and 6].

For a cardinal $\lambda>\omega$, the expression $A=\mathcal{P}_{\lambda}(B)$ can be formalized by claiming that $\lambda$ is a cardinal and $\forall x(x \in A \leftrightarrow(x \subseteq B \wedge|x|<\lambda))$. The clause $|x|<\lambda$ is, on the one hand, equivalent to

$(\exists \alpha \in \lambda) \exists f(f$ is a bijective function from $x$ to $\alpha)$,

which is $\Sigma_{1}$, and on the other hand it is the negation of $\lambda \leq|x|$, hence equivalent to the $\Pi_{1}$ claim that there is no injective function from $\lambda$ to $x$. Therefore, $A=\mathcal{P}_{\lambda}(B)$ is $\Pi_{1}$.

\section{Complexity of categories}

In order to simplify expressions, if $\mathcal{C}$ is a category we shall denote by $X \in \mathcal{C}$ the statement that $X$ is an object of $\mathcal{C}$ and by $f \in \mathcal{C}(X, Y)$ the claim that $X$ and $Y$ are objects of $\mathcal{C}$ and $f$ is a morphism from $X$ to $Y$.

Definition 3.1. For $n \geq 0$, a category $\mathcal{C}$ is called $\boldsymbol{\Sigma}_{n}$-definable (briefly, $\boldsymbol{\Sigma}_{n}$ ) with a set $p$ of parameters if there is a $\Sigma_{n}$ formula $\varphi$ of the language of set theory such that $\varphi(X, Y, Z, f, g, h, i, p)$ is true if and only if $f \in \mathcal{C}(X, Y), g \in \mathcal{C}(Y, Z), h$ is the composite of $f$ and $g$, and $i$ is the identity of $X$.

If a category $\mathcal{C}$ is $\Sigma_{n}$ with a set $p$ of parameters, then there are $\Sigma_{n}$ formulas $\psi_{\mathrm{Ob}}(x, y)$ and $\psi_{\mathrm{Mor}}(x, y, z, t)$ such that $\psi_{\mathrm{Ob}}(X, p)$ is true if and only if $X \in \mathcal{C}$ and $\psi_{\mathrm{Mor}}(X, Y, f, p)$ is true if and only if $f \in \mathcal{C}(X, Y)$. Specifically, from a formula $\varphi$ as in Definition $3.1 \mathrm{we}$ can choose $\psi_{\text {Mor }}(x, y, z, t)$ to be $\exists i \varphi(x, x, y, i, z, z, i, t)$, and next choose $\psi_{\mathrm{Ob}}(x, y)$ to be $\exists z \psi_{\text {Mor }}(x, x, z, y)$.

If $\mathcal{C}$ is $\Sigma_{n}$, then the statement $F=\mathcal{C}(X, Y)$ is formalized with the following $\Sigma_{n} \wedge \Pi_{n}$ formula:

$$
(\forall f \in F) f \in \mathcal{C}(X, Y) \wedge \forall g(g \in \mathcal{C}(X, Y) \rightarrow g \in F) .
$$

We say that a category is $\Pi_{n}$ for $n \geq 0$ if there are $\Pi_{n}$ formulas defining its objects, morphisms, composition and identities. A category will be called $\boldsymbol{\Delta}_{n}$ if it is both $\boldsymbol{\Sigma}_{n}$ and $\Pi_{n}$.

A category is upward absolute for transitive classes if its objects, morphisms, composition and identities can be defined by formulas that are upward absolute for transitive classes. Downward absolute categories are defined in the same way, and a category will be 
called absolute if it is both upward absolute and downward absolute. Thus, $\boldsymbol{\Delta}_{1}$ categories are absolute for transitive classes containing the parameters involved.

If $\mathcal{C}$ is a subcategory of the category of sets, then composition and identities in $\mathcal{C}$ are prescribed by those of sets. Therefore, the complexity of a subcategory of sets is the same if defined as in Definition 3.1 or if simply treated as a class of sets together with a class of functions.

Many important categories which cannot be embedded into Set have nevertheless a complexity in our sense. For example, the homotopy category of simplicial sets cannot be embedded into Set according to [24], and yet it can be defined with a $\Sigma_{2}$ formula, since $\mu$ is a morphism from $X$ to $Y$ if and only if there exists a simplicial map $f$ from $X$ to a fibrant replacement of $Y$ such that $\mu$ is the set of all simplicial maps homotopic to $f$, and composition is defined accordingly (fibrant replacements are discussed in Section 9).

For a category $\mathcal{C}$ and an object $A$ of $\mathcal{C}$, we denote by $(\mathcal{C} \downarrow A)$ the slice category whose objects are pairs $\langle X, f\rangle$ where $f \in \mathcal{C}(X, A)$ and whose morphisms $\langle X, f\rangle \rightarrow\left\langle X^{\prime}, f^{\prime}\right\rangle$ are morphisms $g \in \mathcal{C}\left(X, X^{\prime}\right)$ such that $f=f^{\prime} \circ g$. Dually, the objects of the coslice category $(A \downarrow \mathcal{C})$ are pairs $\langle X, f\rangle$ where $f \in \mathcal{C}(A, X)$, with corresponding morphisms. Both $(\mathcal{C} \downarrow A)$ and $(A \downarrow \mathcal{C})$ are definable with the same complexity as $\mathcal{C}$, with $A$ as an additional parameter. Slice and coslice categories are (non-full) subcategories of the category of arrows $\operatorname{Arr} \mathcal{C}$, whose objects are triples $\langle A, B, f\rangle$ with $f \in \mathcal{C}(A, B)$, which we normally denote by $f: A \rightarrow B$. A morphism $\langle A, B, f\rangle \rightarrow\langle C, D, g\rangle$ is a commutative square

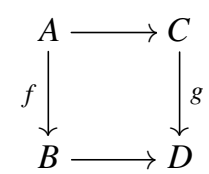

Lemma 3.2. If $\Sigma$ is any signature, then there is a signature $\Sigma^{\prime}$ such that $\operatorname{Arr} \operatorname{Str} \Sigma$ fully embeds into $\operatorname{Str} \Sigma^{\prime}$, and, if $A$ is a $\Sigma$-structure, then there is a signature $\Sigma^{\prime \prime}$ such that $(A \downarrow \operatorname{Str} \Sigma)$ fully embeds into $\operatorname{Str} \Sigma^{\prime \prime}$. In both cases, the embedding preserves complexity.

Proof. Let $S$ be the set of sorts of $\Sigma$. Consider a new set of sorts $S^{\prime}$ with two elements $s^{0}$ and $s^{1}$ for each $s \in S$, and let $\Sigma^{\prime}$ be the $S^{\prime}$-sorted signature with the following operation symbols and relation symbols. The set $\Sigma_{\text {op }}^{\prime}$ has two symbols $\sigma^{0}$ and $\sigma^{1}$ of respective arities $\left\langle\left(s_{i}\right)^{0}: i \in \alpha\right\rangle \rightarrow s^{0}$ and $\left\langle\left(s_{i}\right)^{1}: i \in \alpha\right\rangle \rightarrow s^{1}$ for each symbol $\sigma \in \Sigma_{\text {op }}$ of arity $\left\langle s_{i}: i \in \alpha\right\rangle \rightarrow s$, and an additional symbol $\mu_{s}$ of arity $s^{0} \rightarrow s^{1}$ for each $s \in S$. The set $\Sigma_{\text {rel }}^{\prime}$ has two symbols $\rho^{0}$ and $\rho^{1}$ of respective arities $\left\langle\left(s_{j}\right)^{0}: j \in \beta\right\rangle$ and $\left\langle\left(s_{j}\right)^{1}: j \in \beta\right\rangle$ for each symbol $\rho \in \Sigma_{\text {rel }}$ of arity $\left\langle s_{j}: j \in \beta\right\rangle$.

Then a $\Sigma^{\prime}$-structure is a pair of $\Sigma$-structures $X^{0}$ and $X^{1}$ together with an $S$-sorted function $\mu: X^{0} \rightarrow X^{1}$. Therefore, $\operatorname{Arr} \operatorname{Str} \Sigma$ is canonically isomorphic to the full subcategory of $\operatorname{Str} \Sigma^{\prime}$ whose objects are triples $\left\langle X^{0}, X^{1}, \mu\right\rangle$ for which $\mu$ is a homomorphism of $\Sigma$-structures.

For the second claim, define, as in [2, 1.57(2)], a signature $\Sigma^{\prime \prime}$ by adding to $\Sigma$ a new relation symbol $\rho_{a}$ of arity $s$ for each element $a \in A_{s}$. Then $(A \downarrow \operatorname{Str} \Sigma)$ is canonically isomorphic to the full subcategory of $\operatorname{Str} \Sigma^{\prime \prime}$ whose objects are those $Y \in \operatorname{Str} \Sigma$ for which 
$\left(\rho_{a}\right)_{Y}$ consists of a single element of $Y_{s}$ for each $a \in A_{s}$ and the function $\rho_{Y}: A \rightarrow Y$ given by $\rho_{Y}(a)=\left(\rho_{a}\right)_{Y}$ is a homomorphism of $\Sigma$-structures.

Both embeddings preserve complexity due to their canonical nature. In more detail, suppose given a $\Sigma_{n}$ class $\mathcal{F}$ of objects in $\operatorname{Arr} \operatorname{Str} \Sigma$. Then its image $\mathcal{F}^{\prime}$ in $\operatorname{Str} \Sigma^{\prime}$ is defined as the class of $\Sigma^{\prime}$-structures

$$
\begin{aligned}
& X=\left\langle\left\{X_{s^{0}}: s \in S\right\} \cup\left\{X_{s^{1}}: s \in S\right\},\right. \\
& \left\{\left(\sigma^{0}\right)_{X}: \sigma \in \Sigma_{\text {op }}\right\} \cup\left\{\left(\sigma^{1}\right)_{X}: \sigma \in \Sigma_{\text {op }}\right\} \cup\left\{\left(\mu_{s}\right)_{X}: s \in S\right\}, \\
& \left.\quad\left\{\left(\rho^{0}\right)_{X}: \rho \in \Sigma_{\text {rel }}\right\} \cup\left\{\left(\rho^{1}\right)_{X}: \rho \in \Sigma_{\text {rel }}\right\}\right\rangle
\end{aligned}
$$

for which the triple consisting of

$$
\begin{aligned}
& X^{0}=\left\langle\left\{X_{s^{0}}: s \in S\right\},\left\{\left(\sigma^{0}\right)_{X}: \sigma \in \Sigma_{\mathrm{op}}\right\},\left\{\left(\rho^{0}\right)_{X}: \rho \in \Sigma_{\text {rel }}\right\}\right\rangle, \\
& X^{1}=\left\langle\left\{X_{s^{1}}: s \in S\right\},\left\{\left(\sigma^{1}\right)_{X}: \sigma \in \Sigma_{\mathrm{op}}\right\},\left\{\left(\rho^{1}\right)_{X}: \rho \in \Sigma_{\mathrm{rel}}\right\}\right\rangle,
\end{aligned}
$$

together with the $S$-sorted function $f: X^{0} \rightarrow X^{1}$ given by $f_{s}=\left(\mu_{s}\right)_{X}$ for all $s \in S$ is in the class $\mathcal{F}$. Hence, $\mathcal{F}^{\prime}$ is also $\boldsymbol{\Sigma}_{n}$, and analogously with $\boldsymbol{\Pi}_{n}$.

The argument for $(A \downarrow \operatorname{Str} \Sigma)$ is similar.

Proposition 3.3. If $\Sigma$ is a $\lambda$-ary signature for a regular cardinal $\lambda$, then the following assertions hold:

(a) The category $\operatorname{Str} \Sigma$ of $\Sigma$-structures is $\Pi_{1}$ with parameters $\{\lambda, \Sigma\}$, and it is absolute between transitive classes closed under sequences of length less than $\lambda$ and containing the parameters.

(b) More generally, the category Mod $T$ of models of a theory $T$ in $\mathcal{L}_{\lambda}(\Sigma)$ is $\boldsymbol{\Delta}_{2}$ with parameters $\{\lambda, \Sigma, T\}$, and it is absolute between transitive classes closed under sequences of length less than $\lambda$ and containing the parameters.

Proof. In order to claim that $X$ is a $\Sigma$-structure, we need to formalize the following statement: " $\lambda$ is a regular cardinal, and $\Sigma=\left\langle S, \Sigma_{\text {op }}, \Sigma_{\text {rel }}\right.$, ar $\rangle$ is a $\lambda$-ary signature, and $X=\left\langle\left\{X_{s}: s \in S\right\},\left\{\sigma_{X}: \sigma \in \Sigma_{\text {op }}\right\},\left\{\rho_{X}: \rho \in \Sigma_{\text {op }}\right\}\right\rangle$ is a $\Sigma$-structure". Writing down that $\lambda$ is a regular cardinal is $\Pi_{1}$ by [31, Lemma 13.13], and adding that $\Sigma$ is a $\lambda$-ary signature does not increase complexity. The assertion that $X$ is a $\Sigma$-structure includes the $\Pi_{1}$ formula

$$
\begin{aligned}
\left(\forall \sigma \in \Sigma_{\mathrm{op}}\right)(\forall \alpha \in \lambda)(\forall x)\left[\left[x \text { is a function } \alpha \rightarrow \bigcup_{s \in S} X_{S}\right.\right. \\
\left.\left.\wedge \operatorname{ar}(\sigma)=\left(\left\langle s_{i}: i \in \alpha\right\rangle \rightarrow s\right) \wedge(\forall i \in \alpha) x(i) \in X_{s_{i}}\right] \rightarrow \sigma_{X}(x) \in X_{S}\right] .
\end{aligned}
$$

Hence, the whole statement is $\Pi_{1}$. Similarly, the assertion that $f: X \rightarrow Y$ is a homomorphism of $\Sigma$-structures is $\Pi_{1}$, since we need to impose that $f\left(\sigma_{X}(x)\right)=\sigma_{Y}(f(x))$ for all functions $x: \alpha \rightarrow \bigcup_{s \in S} X_{s}$ with $x(i) \in X_{s_{i}}$ for all $i \in \alpha$, for each operation symbol $\sigma$ of arity $\left\langle s_{i}: i \in \alpha\right\rangle \rightarrow s$. Stating that $f(x) \in \rho_{Y}$ for every $x \in \rho_{X}$ and each relation symbol $\rho$ does not require unbounded quantifiers. 
If $\lambda=\omega$, then we can omit the clause " $\lambda$ is a regular cardinal" and there is only need to quantify over finite sequences in $\bigcup_{s \in S} X_{s}$, which is $\Delta_{1}$, as discussed in Example 2.3.

In order to state that $X$ is a model of a theory $T$, we need to assert that " $X$ is a $\lambda$-ary $\Sigma$-structure, and $T$ is a set of sentences of the language of $\Sigma$, and every sentence of $T$ is satisfied in $X$ ". If $\lambda=\omega$, then this is again $\Delta_{1}$, since satisfaction of sentences of a finitary language in $X$ only depends on finite subsets of $X$. For an arbitrary regular cardinal $\lambda$, the last two clauses are absolute between transitive classes that are closed under sequences of length less than $\lambda$. Hence, by the Reflection Principle, $X$ is a model of $T$ if and only if every $\varphi \in T$ is a sentence of the language of $\Sigma$, and $X$ is a $\Sigma$-structure, and there is a finite fragment $\mathrm{ZFC}^{*}$ of $\mathrm{ZFC}$ such that

$\exists M(M$ is transitive and closed under $<\lambda$-sequences

$$
\left.\wedge\{\lambda, \Sigma, T, X\} \subset M \wedge M \models \wedge \mathrm{ZFC}^{*} \wedge M \models(\forall \varphi \in T) X \models \varphi\right),
$$

which can be replaced with

$\forall M((M$ is transitive and closed under $<\lambda$-sequences

$$
\left.\left.\wedge\{\lambda, \Sigma, T, X\} \subset M \wedge M \models \wedge \mathrm{ZFC}^{*}\right) \rightarrow M \models(\forall \varphi \in T) X \models \varphi\right) .
$$

Since (3.1) is $\Sigma_{2}$ and (3.2) is $\Pi_{2}$, the statement " $X$ is a model of $T$ " is $\Delta_{2}$. And a morphism between models of $T$ is just a homomorphism of $\Sigma$-structures, so the proof of part (b) is complete.

\section{Supporting elementary embeddings}

An elementary embedding of a $\Sigma$-structure $X$ into another $\Sigma$-structure $Y$ (where $X$ and $Y$ can be proper classes) is a function $j: X \rightarrow Y$ that preserves and reflects truth. That is, for every formula $\varphi\left(x_{i}\right)_{i \in I}$ of the language of $\Sigma$ and all $\left\{a_{i}: i \in I\right\}$ in $X$, the sentence $\varphi\left(a_{i}\right)_{i \in I}$ is satisfied in $X$ if and only if $\varphi\left(j\left(a_{i}\right)\right)_{i \in I}$ is satisfied in $Y$.

In what follows, we consider elementary embeddings between structures of the language of set theory. If $j: V \rightarrow M$ is a nontrivial elementary embedding of the universe $V$ of all sets into a transitive class $M$, then its critical point (i.e., the least ordinal moved by $j$ ) is a measurable cardinal. In fact, the existence of a nontrivial elementary embedding of the set-theoretic universe into a transitive class is equivalent to the existence of a measurable cardinal [31, Lemma 17.3].

For a subcategory $\mathcal{C}$ of the category of sets and an elementary embedding $j: V \rightarrow M$, we say that $j$ is supported by $\mathcal{C}$ if, for every object $X$ in $\mathcal{C}$, the set $j(X)$ is also in $\mathcal{C}$ and the restriction function $j\lceil X: X \rightarrow j(X)$ is a morphism in $\mathcal{C}$.

Theorem 4.1. Let $j: V \rightarrow M$ be an elementary embedding with critical point $\kappa$. Let $\Sigma$ be a $\lambda$-ary signature in $V_{\kappa}$ for a regular cardinal $\lambda<\kappa$ such that $M$ is closed under sequences of length less than $\lambda$. If $X$ is a $\Sigma$-structure, then $j(X)$ is also a $\Sigma$-structure and $j\lceil X: X \rightarrow j(X)$ is an elementary embedding of $\Sigma$-structures. 
Proof. First, observe that $j(\lambda)=\lambda$ and hence $\lambda$ is also a regular cardinal in $M$. Next, $j(\Sigma)=\Sigma$ as $\Sigma \in V_{\kappa}$. Therefore, since $j$ is an elementary embedding, if $X$ is a $\Sigma$-structure then $j(X)$ is a $\Sigma$-structure in $M$. It follows that $j(X)$ is also a $\Sigma$-structure in $V$, because, by Proposition 3.3 , being a $\lambda$-ary $\Sigma$-structure is absolute for transitive classes containing $\lambda$ and closed under sequences of length less than $\lambda$.

We next check, by induction on the complexity of formulas of $\mathcal{L}_{\lambda}(\Sigma)$, that $j\lceil X$ is an elementary embedding of $\Sigma$-structures. For atomic formulas, let $\sigma \in \Sigma_{\text {op }}$ be an operation symbol with arity $\left\langle s_{i}: i \in \alpha\right\rangle \rightarrow s$ where $\alpha<\lambda$, so $j(\alpha)=\alpha$. Thus, if $a_{i} \in X_{s_{i}}$ for all $i \in \alpha$, and $a \in X_{s}$, then, since $j$ is elementary, $X \models\left(\sigma_{X}\left(a_{i}\right)_{i \in \alpha}=a\right)$ if and only if

$$
M \models\left(j(X) \models\left(\sigma_{j(X)}\left(j\left(a_{i}\right)\right)_{i \in \alpha}=j(a)\right)\right) .
$$

Since the statement $j(X) \models\left(\sigma_{j(X)}\left(j\left(a_{i}\right)\right)_{i \in \alpha}=j(a)\right)$ is absolute for transitive classes, it holds in $M$ if and only if it holds in $V$, as needed. Relation symbols $\rho \in \Sigma_{\text {rel }}$ are dealt with similarly, and the cases of negation and conjunction are immediate. Thus, it only remains to consider existential formulas. If $X \models \exists x \varphi(x, a)$ for some $a \in X$, then there exists $b \in X$ such that $X \models \varphi(b, a)$. By induction hypothesis, $j(X) \models \varphi(j(b), j(a))$; hence $j(X) \models \exists x \varphi(x, j(a))$. For the converse, observe first that, since $M$ is transitive and closed under sequences of length less than $\lambda$, satisfaction in $j(X)$ of formulas of $\mathcal{L}_{\lambda}(\Sigma)$ is absolute between $M$ and $V$. Hence, if $j(X) \models \exists x \varphi(x, j(a))$ for some $a \in X$, then $M \models(j(X) \models \exists x \varphi(x, j(a)))$ and by elementarity of $j$ we conclude, as needed, that $X \models \exists x \varphi(x, a)$.

Since elementary embeddings of $\Sigma$-structures are homomorphisms, Theorem 4.1 tells us that categories of structures support elementary embeddings with sufficiently large critical point. The following generalization of this fact is a more accurate restatement of [9, Proposition 4.4].

Theorem 4.2. Let $\mathcal{C}$ be a class of $\Sigma$-structures for some $\lambda$-ary signature $\Sigma$, where $\lambda$ is a regular cardinal. Suppose that $\mathcal{C}$ is $\Sigma_{1}$ with a set $p$ of parameters. Let $j: V \rightarrow M$ be an elementary embedding with critical point $\kappa>\lambda$ such that $M$ is closed under sequences of length less than $\lambda$ and $\{p, \Sigma\} \in V_{\kappa}$. If $X \in \mathcal{C}$, then $j(X) \in \mathcal{C}$ and $j\lceil X: X \rightarrow j(X)$ is an elementary embedding of $\Sigma$-structures.

Proof. The proof follows the same steps as the proof of Theorem 4.1, using the fact that $\Sigma_{1}$ formulas are upward absolute to infer that $j(X) \in \mathcal{C}$ for every $X \in \mathcal{C}$.

\section{Vopěnka's principle and supercompact cardinals}

For any two structures $M \subseteq N$ of the language of set theory and $n<\omega$, we write $M \preceq_{n} N$ and say that $M$ is a $\Sigma_{n}$-elementary substructure of $N$ if, for every $\Sigma_{n}$ formula $\varphi\left(x_{1}, \ldots, x_{k}\right)$ and all $a_{1}, \ldots, a_{k} \in M$,

$$
N \models \varphi\left(a_{1}, \ldots, a_{k}\right) \quad \text { if and only if } \quad M \models \varphi\left(a_{1}, \ldots, a_{k}\right) \text {. }
$$


For a cardinal $\lambda$, we denote by $H(\lambda)$ the set of all sets whose transitive closure has cardinality less than $\lambda$. Thus $H(\lambda)$ is a transitive set contained in $V_{\lambda}$, and, if $\lambda$ is strongly inaccessible, then $H(\lambda)=V_{\lambda}$ (see [35, Lemma 6.2]).

A class $C$ of ordinals is unbounded if it contains arbitrarily large ordinals, and it is closed if, for every ordinal $\alpha$, if $\bigcup(C \cap \alpha)=\alpha$ then $\alpha \in C$. The abbreviation $c l u b$ means closed and unbounded. As a consequence of the Reflection Principle [31, Theorem 12.14], for every $n$ there exists a club class of cardinals $\lambda$ such that $H(\lambda) \preceq_{n} V$. In addition, if $\lambda$ is uncountable, then $H(\lambda) \preceq_{1} V$.

In what follows, structures are meant to be sets, not proper classes. We say that $X$ and $Y$ are structures of the same type if they are both $\Sigma$-structures for some signature $\Sigma$. Vopěnka's principle is the following assertion (compare with [2, Ch. 6] or [31, (20.29)]):

VP: For every proper class $\mathcal{C}$ of structures of the same type, there exist distinct $X$ and $Y$ in $\mathcal{C}$ and an elementary embedding of $X$ into $Y$.

This is a statement involving classes. In the language of set theory, one can also formulate VP, but as an axiom schema, that is, an infinite set of axioms; namely, one axiom for each formula $\varphi(x, y)$ of the language of set theory with two free variables, as follows:

$$
\begin{aligned}
\forall x[(\forall y \forall z & ((\varphi(x, y) \wedge \varphi(x, z)) \rightarrow y \text { and } z \text { are structures of the same type }) \\
& \wedge \forall \alpha(\alpha \text { is an ordinal } \rightarrow \exists y(\operatorname{rank}(y)>\alpha \wedge \varphi(x, y)))) \rightarrow \\
& \exists y \exists z(\varphi(x, y) \wedge \varphi(x, z) \wedge y \neq z \wedge \exists e(e: y \rightarrow z \text { is elementary }))] .
\end{aligned}
$$

In this article, VP will be understood as this axiom schema, and similarly with the variants of VP defined below.

In the statement of VP, the requirement that there is an elementary embedding between two distinct structures is sometimes replaced by the requirement that there is a nontrivial elementary embedding between two possibly equal structures. It follows from [14] that it is consistent with ZFC to assume that the two formulations are equivalent. Equivalence can be proved using rigid graphs, as in $[2, \S 6 . \mathrm{A}]$, although this seems to require the use of global choice.

The theory ZFC+VP is very strong. It implies, for instance, that the class of extendible cardinals is stationary; that is, every club proper class contains an extendible cardinal [37]. The consistency of ZFC + VP follows from that of ZFC plus the existence of an almosthuge cardinal (see [31] or [33]).

If $\lambda$ and $v$ are cardinals, we denote by $v^{<\lambda}$ the union of $v^{\alpha}$ for all $\alpha<\lambda$. If $f: A \rightarrow B$ is a homomorphism of structures and $M$ is any set, when we write that $f \in M$ we mean that $A, B \in M$ and $\{(a, f(a)): a \in A\} \in M$.

Theorem 5.1. Let $\mathcal{C}$ be a full subcategory of $\Sigma$-structures definable by a $\Sigma_{1}$ formula with a set $p$ of parameters for some $\lambda$-ary signature $\Sigma$. Let $\kappa$ be a regular cardinal bigger than $\lambda$ such that $\{p, \Sigma\} \in H(\kappa)$ and with the property that $v^{<\lambda}<\kappa$ for all $v<\kappa$. Then the following hold: 
(a) For every homomorphism $g: A \rightarrow Y$ of $\Sigma$-structures with $A \in H(\kappa)$ and $Y \in \mathcal{C}$ there is a homomorphism $f: A \rightarrow X$ with $X \in \mathcal{C} \cap H(\kappa)$ and a commutative triangle

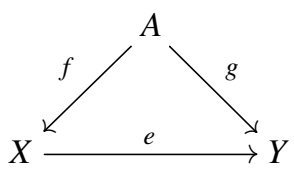

where $e$ is an elementary embedding.

(b) Every object $Y \in \mathcal{C}$ has a subobject $X \in \mathcal{C} \cap H(\kappa)$.

Proof. We only have to prove (a), since (b) then follows with $A=\emptyset$. Note that every elementary embedding of $\Sigma$-structures is an injective homomorphism and, since $\mathcal{C}$ is a full subcategory, $e: X \rightarrow Y$ is in $\mathcal{C}$, so $X$ is a subobject of $Y$, since, in a subcategory of sets, every injective morphism is a monomorphism (see [1, Proposition 7.37]).

Thus, suppose that $\mathcal{C}$, viewed as a class, is definable as $\mathcal{C}=\{x: \varphi(x, p)\}$, where $\varphi$ is $\Sigma_{1}$ and $p \in H(\kappa)$. Given $g: A \rightarrow Y$ with $A \in H(\kappa)$ and $Y \in \mathcal{C}$, let $\mu$ be a regular cardinal bigger than $\kappa$ such that $Y \in H(\mu)$ and such that $H(\mu) \models \varphi(Y, p)$.

In this situation, the Löwenheim-Skolem Theorem implies the existence of an elementary substructure $\langle N, \in\rangle$ of $\langle H(\mu), \in\rangle$ of cardinality smaller than $\kappa$ and closed under sequences of length less than $\lambda$ (here we use the assumption that $v^{<\lambda}<\kappa$ for all $\nu<\kappa$ ) such that $g \in N$ and with the transitive closure of $\{p, \Sigma, A\}$ contained in $N$. By elementarity, $g$ is a homomorphism of $\Sigma$-structures in $N$ and $N \models \varphi(Y, p)$.

Let $M$ be the transitive collapse of $N$, and let $j: M \rightarrow N$ be the isomorphism given by the collapse, that is, $j$ is inverse to the function $\pi(x)=\{\pi(z): z \in x\}$ (see [31,6.13]). Since $N$ is closed under sequences of length less than $\lambda$, so is $M$, and the critical point of $j$ is greater than or equal to $\lambda$. And since $N$ contains the transitive closure of $\{p, \Sigma, A\}$, we have $\pi(p)=p, \pi(\Sigma)=\Sigma$ and $\pi(A)=A$. Moreover, the restriction $j\lceil A$ is the identity.

Now let $X \in M$ be such that $j(X)=Y$ and let $f: A \rightarrow X$ be such that $j(f)=g$. Then $X \in H(\kappa)$ since $|M|<\kappa$ and $M$ is transitive. Since $j$ is an isomorphism and $j(p)=p$, we infer that $M \models \varphi(X, p)$, and hence, as $\Sigma_{1}$ formulas are upward absolute for transitive classes, we conclude that $X \in \mathcal{C}$ in $V$. Since $j(\Sigma)=\Sigma$ and $M$ and $N$ are closed under sequences of length less than $\lambda$, the object $X$ is a $\Sigma$-structure and, since $j$ is an isomorphism, the restriction $e=j\lceil X$ is an elementary embedding, hence a homomorphism of $\Sigma$-structures. Moreover, $f$ is also a homomorphism and the triangle commutes since $f$ has been defined so that $g(a)=j(f(a))$ for all $a \in A$.

Recall that a cardinal $\kappa$ is called $\lambda$-supercompact if there is an elementary embedding $j: V \rightarrow M$ with $M$ transitive and with critical point $\kappa$ such that $j(\kappa)>\lambda$ and $M$ is closed under $\lambda$-sequences. Note that it then follows that $H(\lambda) \in M$. A cardinal $\kappa$ is supercompact if it is $\lambda$-supercompact for all ordinals $\lambda$.

The following theorem is an upgraded version of [9, Theorem 4.5], where a similar result was proved for absolute classes. 
Theorem 5.2. Let $\mathcal{C}$ be a full subcategory of $\Sigma$-structures definable by a $\Sigma_{2}$ formula with a set $p$ of parameters. Suppose that there exists a supercompact cardinal $\kappa$ bigger than the rank of $p$ and $\Sigma$. Then the following hold:

(a) For every homomorphism $g: A \rightarrow Y$ of $\Sigma$-structures with $A \in V_{\kappa}$ and $Y \in \mathcal{C}$ there is a homomorphism $f: A \rightarrow X$ with $X \in \mathcal{C} \cap V_{\kappa}$ and an elementary embedding $e: X \rightarrow Y$ with $e \circ f=g$.

(b) Every object $Y \in \mathcal{C}$ has a subobject $X \in \mathcal{C} \cap V_{\kappa}$.

Proof. As with Theorem 5.1, we only have to prove (a), since (b) follows by taking $A=\emptyset$. Suppose that $\kappa$ is a supercompact cardinal for which $\{p, \Sigma, A\} \in V_{\kappa}$. Then, since $\kappa$ is strongly inaccessible, we have $V_{\kappa}=H(\kappa)$ and, since $\kappa$ is regular, it is bigger than the supremum of the ordinals of the arities of all the operation symbols and relation symbols of $\Sigma$, so $\Sigma$ is $\kappa$-ary.

Given a homomorphism $g: A \rightarrow Y$ with $Y \in \mathcal{C}$, let $\mu$ be a cardinal bigger than $\kappa$ such that $Y \in H(\mu)$ and $H(\mu) \preceq_{2} V$. Let $j: V \rightarrow M$ be an elementary embedding with $M$ transitive and critical point $\kappa$, such that $j(\kappa)>\mu$ and $M$ is closed under $\mu$-sequences. Then $j(A)=A$ since $A$ is in $H(\kappa)$, and $g$ and the restriction $j\lceil Y: Y \rightarrow j(Y)$ are in $M$ because $A, Y \in M$ and $M$ is closed under $\mu$-sequences. In addition, $g: A \rightarrow Y$ is a homomorphism of $\Sigma$-structures in $M$, since, by Proposition 3.3, being a homomorphism of $\kappa$-ary $\Sigma$-structures is absolute for transitive classes containing $\Sigma$ and closed under sequences of length less than $\kappa$. Moreover, by Theorem 4.1, since $\Sigma \in V_{\kappa}$, the restriction $j\lceil Y: Y \rightarrow j(Y)$ is an elementary embedding of $\Sigma$-structures.

Since being a cardinal is $\Pi_{1}$ and hence downward absolute, $\mu$ is a cardinal in $M$, and this implies that $H(\mu)$ in the sense of $M$ coincides with $H(\mu)$. It follows that $H(\mu) \preceq_{1} M$, since every $\Sigma_{1}$ sentence $\psi$ which holds in $M$ also holds in $V$ (as $\Sigma_{1}$ sentences are upward absolute) and therefore $\psi$ holds in $H(\mu)$ because $H(\mu) \preceq_{2} V$. Hence, $\Sigma_{2}$ formulas are upward absolute between $H(\mu)$ and $M$. Since $H(\mu) \preceq_{2} V$ and the class $\mathcal{C}$ is defined by a $\Sigma_{2}$ formula $\varphi(x, y)$, we see that $H(\mu) \models \varphi(Y, p)$ and thus $M=\varphi(Y, p)$.

Now $\operatorname{rank}(Y)<\mu<j(\kappa)$ in $V$ and also in $M$. Thus, as witnessed by $g: A \rightarrow Y$, in $M$ there exists a homomorphism $f: A \rightarrow X$ of $\Sigma$-structures such that $\operatorname{rank}(X)<$ $j(\kappa)$ and $\varphi(X, p)$ holds, and there is an elementary embedding $e: X \rightarrow j(Y)$ such that $e \circ f=j(g)$. By elementarity of $j$, the corresponding statement is true in $V$; that is, there exists a homomorphism of $\Sigma$-structures $f: A \rightarrow X$ such that $\operatorname{rank}(X)<\kappa$ and $\varphi(X, p)$ holds, so $X \in \mathcal{C}$, and there is an elementary embedding $e: X \rightarrow Y$ with $e \circ f=g$, as we wanted to prove.

Theorem 5.2 tells us that the existence of arbitrarily large supercompact cardinals implies that VP holds for $\boldsymbol{\Sigma}_{2}$ proper classes. The following theorem yields a strong converse of this fact.

Theorem 5.3. Suppose that, for every $\boldsymbol{\Delta}_{2}$ proper class $\mathcal{C}$ of structures in the language of set theory with one additional constant symbol, there exist distinct $X$ and $Y$ in $\mathcal{C}$ and an elementary embedding of $X$ into $Y$. Then there exists a proper class of supercompact cardinals. 
Proof. Let $\xi$ be any ordinal and suppose, towards a contradiction, that there are no supercompact cardinals bigger than $\xi$. Then the class function $F$ given as follows is well defined on ordinals $\zeta>\xi: F(\zeta)$ equals the least cardinal $\lambda>\zeta$ such that no cardinal $\kappa$ such that $\xi<\kappa \leq \zeta$ is $\lambda$-supercompact. Since the assertion " $\zeta$ is $\lambda$-supercompact" is $\Delta_{2}$ in ZFC (see [33, §22]), $F$ is $\boldsymbol{\Delta}_{2}$-definable with $\xi$ as a parameter. Let

$$
C_{0}=\{\alpha: \alpha \text { is a limit ordinal, } \xi<\alpha \text {, and } \forall \zeta(\xi<\zeta<\alpha \rightarrow F(\zeta)<\alpha)\}
$$

Then $C_{0}$ is a club class $\boldsymbol{\Delta}_{2}$-definable with $\xi$ as a parameter.

Fix a rigid binary relation (i.e., a rigid graph) $R$ on $\xi+1$ (see [41]). For each ordinal $\alpha$, let $\lambda_{\alpha}$ be the least element of $C_{0}$ greater than $\lambda$. The proper class $\mathcal{C}=\left\{\left\langle V_{\lambda_{\alpha}+2}, \in,\langle\alpha, R\rangle\right\rangle\right.$ : $\alpha>\xi\}$ is $\boldsymbol{\Delta}_{2}$-definable with $R$ as a parameter. By our assumption, there exist $\alpha<\beta$ greater than $\xi$ and an elementary embedding

$$
j:\left\langle V_{\lambda_{\alpha}+2}, \in,\langle\alpha, R\rangle\right\rangle \rightarrow\left\langle V_{\lambda_{\beta}+2}, \in,\langle\beta, R\rangle\right\rangle
$$

Since $j$ must send $\alpha$ to $\beta$, it is not the identity. Hence, by Kunen's Theorem ([31, Theorem 17.7], [34]), we have $\lambda_{\alpha}<\lambda_{\beta}$. Let $\kappa \leq \alpha$ be the critical point of $j$. Then, as in [37, Lemma 2], it follows that $\kappa$ is $\lambda_{\alpha}$-supercompact. But this is impossible, since $F(\kappa)<\lambda_{\alpha}$ because $\lambda_{\alpha} \in C_{0}$.

In order to summarize what we have proved so far, we introduce some useful notation. Let $\Gamma$ be one of $\Sigma_{n}, \Pi_{n}, \Delta_{n}, \Sigma_{n} \wedge \Pi_{n}$ or $\Sigma_{n}, \Pi_{n}, \Delta_{n}, \Sigma_{n} \wedge \Pi_{n}$, for any $n$. For an infinite cardinal $\kappa$ and a signature $\Sigma \in H(\kappa)$, we write:

$\operatorname{VP}^{\Sigma}(\Gamma):$ For every $\Gamma$ proper class $\mathcal{C}$ of $\Sigma$-structures, there exist distinct $X$ and $Y$ in $\mathcal{C}$ and an elementary embedding of $X$ into $Y$.

$\operatorname{SVP}_{\kappa}^{\Sigma}(\Gamma):$ For every proper class $\mathcal{C}$ of $\Sigma$-structures admitting a $\Gamma$ definition whose parameters, if any, are in $H(\kappa)$, and for every $Y \in \mathcal{C}$, there exists $X \in \mathcal{C} \cap H(\kappa)$ and an elementary embedding of $X$ into $Y$.

If $\Sigma$ is omitted from the notation, we mean that the corresponding statement holds for all admissible signatures. Thus, $\operatorname{VP}(\Gamma)$ means $\operatorname{VP}^{\Sigma}(\Gamma)$ for all $\Sigma$, while $\operatorname{SVP}_{\kappa}(\Gamma)$ means $\operatorname{SVP}_{\kappa}^{\Sigma}(\Gamma)$ for every $\Sigma \in H(\kappa)$.

Even though $\operatorname{SVP}_{\kappa}^{\Sigma}(\Gamma)$ is an apparently stronger statement than $\operatorname{VP}^{\Sigma}(\Gamma)$ (hence the notation SVP), in the case of $\Sigma_{2}$ classes of structures they turn out to be equivalent, as we next prove.

Corollary 5.4. The following statements are equivalent:

(1) $\operatorname{SVP}_{\kappa}\left(\Sigma_{2}\right)$ holds for a proper class of cardinals $\kappa$.

(2) $\operatorname{VP}\left(\boldsymbol{\Sigma}_{2}\right)$ holds.

(3) $\operatorname{VP}^{\Sigma}\left(\boldsymbol{\Delta}_{2}\right)$ holds if $\Sigma$ is the signature of the language of set theory with one additional constant symbol.

(4) There exists a proper class of supercompact cardinals. 
Proof. In order to check that (1) $\Rightarrow(2)$, suppose that (1) is true, and let $\Sigma$ be any signature. Let $\mathcal{C}$ be any proper class of $\Sigma$-structures defined by a $\Sigma_{2}$ formula with parameters, and let $\kappa$ be bigger than the ranks of the parameters and such that $\operatorname{SVP}_{\kappa}^{\Sigma}\left(\Sigma_{2}\right)$ holds. Since $\mathcal{C}$ is a proper class, we may choose $Y$ of rank bigger than $\kappa$, so any $X \in \mathcal{C} \cap H(\kappa)$ will necessarily be distinct from $Y$. Hence, there exist distinct $X$ and $Y$ such that $X$ is elementarily embeddable into $Y$, so $\operatorname{VP}^{\Sigma}\left(\Sigma_{2}\right)$ holds, as needed. The implication $(2) \Rightarrow(3)$ is trivial, and Theorem 5.3 implies that $(3) \Rightarrow(4)$. Finally, to see that $(4) \Rightarrow(1)$, let $\xi$ be any cardinal and pick a supercompact cardinal $\kappa>\xi$. Since $H(\kappa)=V_{\kappa}$, Theorem 5.2 tells us that $\operatorname{SVP}_{\kappa}\left(\Sigma_{2}\right)$ holds.

The following is a corresponding version without parameters, with the same proof:

Corollary 5.5. The following statements are equivalent:

(1) $\operatorname{SVP}_{\kappa}\left(\Sigma_{2}\right)$ holds for some cardinal $\kappa$.

(2) $\operatorname{VP}\left(\Sigma_{2}\right)$ holds.

(3) $\operatorname{VP}^{\Sigma}\left(\Delta_{2}\right)$ holds if $\Sigma$ is the signature of the language of set theory.

(4) There exists a supercompact cardinal.

\section{Vopěnka's principle and extendible cardinals}

For cardinals $\kappa<\lambda$, we say that $\kappa$ is $\lambda$-extendible if there is an elementary embedding $j: V_{\lambda} \rightarrow V_{\mu}$ for some $\mu$, with critical point $\kappa$ and with $j(\kappa)>\lambda$. A cardinal $\kappa$ is called extendible if it is $\lambda$-extendible for all cardinals $\lambda>\kappa$. As shown in [31, 20.24], extendible cardinals are supercompact. See [31] or [33] for related background.

For each $n<\omega$, let $C(n)$ denote the club proper class of infinite cardinals $\kappa$ that are $\Sigma_{n}$-correct in $V$, that is, $V_{\kappa} \preceq_{n} V$. Since the satisfaction relation $\models_{n}$ for $\Sigma_{n}$ sentences (which is, in fact, a proper class) is $\Sigma_{n}$-definable for $n \geq 1$ [33, §0.2], it follows that, for $n \geq 1$, the class $C(n)$ is $\Pi_{n}$. To see this, note first that $C(0)$ is the class of all infinite cardinals, and therefore it is $\Pi_{1}$-definable. For $\kappa$ an infinite cardinal, $\kappa \in C(1)$ if and only if $\kappa$ is an uncountable cardinal and $V_{\kappa}=H(\kappa)$, which implies that $C(1)$ is $\Pi_{1}$-definable. In general, for $n \geq 1$ and for any infinite cardinal $\kappa$, we have $V_{\kappa} \preceq_{n+1} V$ if and only if

$$
\kappa \in C(n) \wedge\left(\forall \varphi(x) \in \Sigma_{n+1}\right)\left(\forall a \in V_{\kappa}\right)\left(\models_{n+1} \varphi(a) \rightarrow V_{\kappa} \models \varphi(a)\right),
$$

which is a $\Pi_{n+1}$ formula showing that $C(n+1)$ is $\Pi_{n+1}$-definable.

We shall use the following new strong form of extendibility.

Definition 6.1. For $C$ a club proper class of cardinals and $\kappa<\lambda$ in $C$, we say that $\kappa$ is $\lambda$-C-extendible if there is an elementary embedding $j: V_{\lambda} \rightarrow V_{\mu}$ for some $\mu \in C$, with critical point $\kappa$, such that $j(\kappa)>\lambda$ and $j(\kappa) \in C$. We say that a cardinal $\kappa$ in $C$ is $C$-extendible if it is $\lambda$-C -extendible for all $\lambda$ in $C$ greater than $\kappa$.

Note that, for all $n$, if $\kappa$ is $C(n)$-extendible, then $\kappa$ is extendible. Therefore, a cardinal is $C(0)$-extendible if and only if it is extendible. 
Proposition 6.2. Every extendible cardinal is C(1)-extendible.

Proof. Suppose that $\kappa$ is extendible and $\lambda \in C(1)$ is greater than $\kappa$. Note that the existence of an extendible cardinal implies the existence of a proper class of inaccessible cardinals, as the image of $\kappa$ under any elementary embedding $j: V_{\lambda} \rightarrow V_{\mu}$, with critical point $\kappa$ and $\lambda$ a cardinal, is always an inaccessible cardinal in $V$. So we can pick an inaccessible cardinal $\lambda^{\prime} \geq \lambda$. Let $j^{\prime}: V_{\lambda^{\prime}} \rightarrow V_{\mu^{\prime}}$ be an elementary embedding with critical point $\kappa$ and such that $j^{\prime}(\kappa)>\lambda^{\prime}$. Since $V_{\lambda^{\prime}}=H\left(\lambda^{\prime}\right)$, it follows by elementarity of $j^{\prime}$ that $V_{\mu^{\prime}}=$ $H\left(\mu^{\prime}\right)$. Hence, $\mu^{\prime} \in C(1)$.

Let us see that $j=j^{\prime} \uparrow V_{\lambda}: V_{\lambda} \rightarrow V_{j^{\prime}(\lambda)}$ witnesses the $\lambda$-C(1)-extendibility of $\kappa$. We only need to check that $\mu=j^{\prime}(\lambda) \in C(1)$. But since $V_{\lambda} \preceq_{1} V_{\lambda^{\prime}}$, it follows by elementarity of $j^{\prime}$ that $V_{\mu} \preceq_{1} V_{\mu^{\prime}}$. Hence, since $\mu^{\prime} \in C(1)$, also $\mu \in C(1)$.

Hence, a cardinal is $C(1)$-extendible if and only if it is extendible. Let us also observe that, if there exists a $C(n+2)$-extendible cardinal for $n \geq 1$, then there exists a proper class of $C(n)$-extendible cardinals (see [7]).

Lemma 6.3. If $\kappa$ is $C(n)$-extendible, then $\kappa \in C(n+2)$.

Proof. By induction on $n$. For $n=0$, since $\kappa \in C(1)$, we only need to show that if $\exists x \varphi(x)$ is a $\Sigma_{2}$ sentence, where $\varphi$ is $\Pi_{1}$ and has parameters in $V_{\kappa}$, that holds in $V$, then it holds in $V_{\kappa}$. So suppose that $a$ is such that $\varphi(a)$ holds in $V$. Let $\lambda \in C(n)$ be greater than $\kappa$ and with $a \in V_{\lambda}$, and let $j: V_{\lambda} \rightarrow V_{\mu}$ be elementary, with critical point $\kappa$ and with $j(\kappa)>\lambda$. Then $V_{j(\kappa)} \models \varphi(a)$, and so, by elementarity, $V_{\kappa} \models \exists x \varphi(x)$.

Now suppose that $\kappa$ is $C(n)$-extendible and $\exists x \varphi(x)$ is a $\Sigma_{n+2}$ sentence, where $\varphi$ is $\Pi_{n+1}$ and has parameters in $V_{\kappa}$. If $\exists x \varphi(x)$ holds in $V_{\kappa}$, then, since by the induction hypothesis $\kappa \in C(n+1)$, we infer that $\exists x \varphi(x)$ holds in $V$. Now suppose that $a$ is such that $\varphi(a)$ holds in $V$. Let $\lambda \in C(n)$ be greater than $\kappa$ and such that $a \in V_{\lambda}$, and let $j: V_{\lambda} \rightarrow V_{\mu}$ be elementary with critical point $\kappa$ and with $j(\kappa)>\lambda$. Then, since $j(\kappa) \in C(n)$, we have $V_{j(\kappa)} \models \varphi(a)$, and so, by elementarity, $V_{\kappa} \models \exists x \varphi(x)$.

Theorem 6.4. For $n \geq 1$, if $\kappa$ is a $C(n)$-extendible cardinal, then $\operatorname{SVP}_{\kappa}\left(\boldsymbol{\Sigma}_{n+2}\right)$ holds.

Proof. Fix a $\Sigma_{n+2}$ formula $\exists x \varphi(x, y, z)$, where $\varphi$ is $\Pi_{n+1}$, such that

$$
\mathcal{C}=\{Y: \exists x \varphi(x, Y, p)\}
$$

is a proper class of structures of the same type for some set $p \in V_{\kappa}$. Fix $Y \in \mathcal{C}$ and let $\lambda \in C(n+2)$ be greater than $\kappa$ and the ranks of $p$ and $Y$. Thus, $V_{\lambda} \models \exists x \varphi(x, B, p)$. Let $j: V_{\lambda} \rightarrow V_{\mu}$, for some cardinal $\mu \in C(n)$, be an elementary embedding with critical point $\kappa$, with $j(\kappa)>\lambda$ and $j(\kappa) \in C(n)$. Note that both $Y$ and $j\lceil Y: Y \rightarrow j(Y)$ are in $V_{\mu}$. Since $\kappa, \lambda \in C(n+2)$ by Lemma 6.3, and $\kappa<\lambda$, we have $V_{\kappa} \preceq_{n+2} V_{\lambda}$. It follows that $V_{j(\kappa)} \preceq_{n+2} V_{\mu}$. Indeed, the following holds:

$$
V_{\lambda} \models\left(\forall x \in V_{\kappa}\right)\left(\forall \theta \in \Sigma_{n+2}\right)\left(V_{\kappa} \models \theta(x) \leftrightarrow \models_{n+2} \theta(x)\right) .
$$

Hence, by elementarity,

$$
V_{\mu} \models\left(\forall x \in V_{j(\kappa)}\right)\left(\forall \theta \in \Sigma_{n+2}\right)\left(V_{j(\kappa)} \models \theta(x) \leftrightarrow \models_{n+2} \theta(x)\right),
$$


which implies that $V_{j(\kappa)} \preceq_{n+2} V_{\mu}$. Since $j(\kappa) \in C(n)$, we have $V_{\lambda} \preceq_{n+1} V_{j(\kappa)}$, and therefore $V_{\lambda} \preceq_{n+1} V_{\mu}$. It follows that $V_{\mu} \models \exists x \varphi(x, Y, b)$.

Thus, in $V_{\mu}$ it is true that there exists $X \in V_{j(\kappa)}$ such that $X \in \mathcal{C}$, namely $Y$, and there exists an elementary embedding $e: X \rightarrow j(Y)$, namely $j \mid Y$. Therefore, by elementarity of $j$, the same is true in $V_{\lambda}$; that is, there exists $X \in V_{\kappa}$ such that $X \in \mathcal{C}$, and there exists an elementary embedding $e: X \rightarrow Y$. Since $\lambda \in C(n+2)$, we have $X \in \mathcal{C}$ and we are done.

Corollary 6.5. If $\kappa$ is an extendible cardinal, then $\operatorname{SVP}_{\kappa}\left(\boldsymbol{\Sigma}_{3}\right)$ holds.

Proof. This is the assertion of Theorem 6.4 for $n=1$.

Corollary 6.6. Let $\mathcal{C}$ be a full subcategory of $\Sigma$-structures definable by a $\Sigma_{n+2}$ formula with a set $p$ of parameters, where $n \geq 1$. Suppose that there exists a $C(n)$-extendible cardinal $\kappa$ bigger than the rank of $p$ and $\Sigma$. Then the following hold:

(a) For every homomorphism $g: A \rightarrow Y$ of $\Sigma$-structures with $A \in V_{\kappa}$ and $Y \in \mathcal{C}$ there is a homomorphism $f: A \rightarrow X$ with $X \in \mathcal{C} \cap V_{\kappa}$ and an elementary embedding $e: X \rightarrow Y$ with $e \circ f=g$.

(b) Every object $Y \in \mathcal{C}$ has a subobject $X \in \mathcal{C} \cap V_{\kappa}$.

Proof. Part (b) is a consequence of Theorem 6.4, and part (a) is a more general variant proved as in Theorem 5.2.

The following theorem yields a converse to Theorem 6.4.

Theorem 6.7. Let $n \geq 1$, and suppose that $\mathrm{VP}^{\Sigma}\left(\Sigma_{n+1} \wedge \Pi_{n+1}\right)$ holds when $\Sigma$ is the signature of the language of set theory with finitely many additional 1-ary relation symbols. Then there exists a $C(n)$-extendible cardinal.

Proof. Suppose, to the contrary, that there is no $C(n)$-extendible cardinal. Then the class function $F$ on ordinals given by defining $F(\zeta)$ to be the least $\lambda>\zeta$ such that $\lambda \in C(n)$ and $\zeta$ is not $\lambda-C(n)$-extendible is well defined.

For $\lambda \in C(n)$, the relation " $\zeta$ is $\lambda$-C(n)-extendible" is $\Sigma_{n+1}$, for it holds if and only if $\zeta \in C(n)$ and

$\exists \mu \exists j: V_{\lambda} \rightarrow V_{\mu}(j$ is elementary $\wedge \operatorname{cp}(j)=\zeta \wedge j(\zeta)>\lambda \wedge \mu, j(\zeta) \in C(n))$,

where $\operatorname{cp}(j)$ denotes the critical point of $j$. Hence $F$ is $\Sigma_{n+1} \wedge \Pi_{n+1}$.

Let $C=\{\alpha: \alpha$ is a limit ordinal and $(\forall \zeta<\alpha) F(\zeta)<\alpha\}$. So, $C$ is a $\Sigma_{n+1} \wedge \Pi_{n+1}$ closed unbounded proper class.

For each ordinal $\alpha$, let $\lambda_{\alpha}$ be the first limit point of $D=C \cap C(n)$ above $\alpha$. Note that the class function $f$ on ordinals such that $f(\alpha)=\lambda_{\alpha}$ is $\left(\Sigma_{n+1} \wedge \Pi_{n+1}\right)$-definable. Now let

$$
\mathcal{C}=\left\{\left\langle V_{\lambda_{\alpha}}, \in, \alpha, \lambda_{\alpha}, C \cap \alpha+1\right\rangle: \alpha \in D\right\} .
$$

We claim that $\mathcal{C}$ is $\left(\Sigma_{n+1} \wedge \Pi_{n+1}\right)$-definable. To see this, observe that $X \in \mathcal{C}$ if and only if $X=\left\langle X_{0}, X_{1}, X_{2}, X_{3}, X_{4}\right\rangle$, where
(1) $X_{2} \in C$;
(2) $X_{3}=\lambda_{X_{2}}$
(3) $X_{0}=V_{X_{3}}$;
(4) $X_{1}=\in \mid X_{0}$;
(5) $X_{4}=C \cap X_{2}+1$. 
We have already seen that (1) and (2) are $\Sigma_{n+1} \wedge \Pi_{n+1}$ expressible. And so are (3) and (4). As for (5), note that $X_{4}=C \cap \alpha+1$ holds in $V$ if and only if it holds in $V_{X_{3}}$.

So $\mathcal{C}$ is a $\Sigma_{n+1} \wedge \Pi_{n+1}$ proper class of structures of the same type in the language of set theory with three additional relation symbols. By our assumption, there are $\alpha<\beta$ in $D$ and an elementary embedding

$$
j:\left\langle V_{\lambda_{\alpha}}, \in, \alpha, \lambda_{\alpha}, C \cap \alpha+1\right\rangle \rightarrow\left\langle V_{\lambda_{\beta}}, \in, \beta, \lambda_{\beta}, C \cap \beta+1\right\rangle .
$$

Since $j$ sends $\alpha$ to $\beta$, it is not the identity. Let $\kappa$ be the critical point of $j$.

Since $\alpha \in C$, we have $\kappa<F(\kappa)<\alpha$. Thus, $j\left\lceil V_{F(\kappa)}: V_{F(\kappa)} \rightarrow V_{j(F(\kappa))}\right.$ is elementary, with critical point $\kappa$.

We claim that $\kappa \in D$. Otherwise, $\gamma=\sup (D \cap \kappa)<\kappa$. Let $\delta$ be the least ordinal in $D$ greater than $\gamma$ with $\kappa<\delta<\lambda_{\alpha}$. Since $\delta$ is definable from $\gamma$ in the structure $\left\langle V_{\lambda_{\alpha}}, \in, \alpha, C \cap \alpha+1\right\rangle$, and since $j(\gamma)=\gamma$, we must also have $j(\delta)=\delta$. But then we conclude that $j\left\lceil V_{\delta+2}: V_{\delta+2} \rightarrow V_{\delta+2}\right.$ is an elementary embedding, contradicting Kunen's Theorem [34].

By elementarity, $j(\kappa) \in C(n)$. Moreover, since $F(\kappa) \in C(n)$ and $\lambda_{\beta} \in C(n)$, we have $j(F(\kappa)) \in C(n)$. Since $\kappa \in C$, by elementarity we also have $j(\kappa) \in C$. Hence, $j(\kappa)>F(\kappa)$. This shows that $j\left\lceil V_{F(\kappa)}\right.$ witnesses that $\kappa$ is $F(\kappa)-C(n)$-extendible, and this contradicts the definition of $F$.

The proof of Theorem 6.7 easily generalizes to the boldface case (see the proof of Theorem 5.3), namely if $\operatorname{VP}\left(\boldsymbol{\Sigma}_{n+1} \wedge \boldsymbol{\Pi}_{n+1}\right)$ holds, then there is a proper class of $C(n)$-extendible cardinals. In fact it is sufficient to assume that $\operatorname{VP}^{\Sigma}\left(\Sigma_{n+1} \wedge \boldsymbol{\Pi}_{n+1}\right)$ holds when $\Sigma$ is the signature of the language of set theory with a finite number of additional 1-ary relation symbols.

The following corollaries summarize our results in this section.

Corollary 6.8. The following statements are equivalent for $n \geq 1$ :

(1) $\operatorname{SVP}_{\kappa}\left(\Sigma_{n+2}\right)$ holds for some cardinal $\kappa$.

(2) $\operatorname{VP}\left(\Sigma_{n+1} \wedge \Pi_{n+1}\right)$ holds.

(3) $\operatorname{VP}^{\Sigma}\left(\Sigma_{n+1} \wedge \Pi_{n+1}\right)$ holds when $\Sigma$ is the signature of the language of set theory with a finite number of additional 1-ary relation symbols.

(4) There exists a $C(n)$-extendible cardinal.

Corollary 6.9. The following statements are equivalent:

(1) For every $n, \operatorname{SVP}_{\kappa}\left(\boldsymbol{\Sigma}_{n}\right)$ holds for a proper class of cardinals $\kappa$.

(2) For every $n, \operatorname{SVP}_{\kappa}\left(\Sigma_{n}\right)$ holds for some cardinal $\kappa$.

(3) $\operatorname{VP}\left(\Sigma_{n}\right)$ holds for all $n$.

(4) $\operatorname{VP}^{\Sigma}\left(\Sigma_{n}\right)$ holds for all $n$ when $\Sigma$ is the signature of the language of set theory with a finite number of additional 1-ary relation symbols.

(5) There exists a $C(n)$-extendible cardinal for every $n$.

(6) There exists a proper class of $C(n)$-extendible cardinals for every $n$.

(7) Vopěnka's principle holds. 


\section{Accessible categories}

A category is small if its objects form a set, and essentially small if the isomorphism classes of its objects form a set.

Let $\lambda$ be a regular cardinal. A nonempty category $\mathcal{K}$ is called $\lambda$-filtered if, given any set $\left\{k_{i}\right\}_{i \in I}$ of objects in $\mathcal{K}$ where $|I|<\lambda$, there is an object $k \in \mathcal{K}$ and a morphism $k_{i} \rightarrow k$ for each $i \in I$, and, moreover, given any set $\left\{f_{j}: k \rightarrow k^{\prime}\right\}_{j \in J}$ of parallel arrows between any two objects where $|J|<\lambda$, there is a morphism $g: k^{\prime} \rightarrow k^{\prime \prime}$ such that $g \circ f_{j}$ is the same morphism for all $j \in J$. If $\mathcal{C}$ is any category, a functor $D: \mathcal{K} \rightarrow \mathcal{C}$ where $\mathcal{K}$ is a $\lambda$-filtered small category is called a $\lambda$-filtered diagram, and, if $D$ has a colimit $L$, then $L$ is called a $\lambda$-filtered colimit. For example, every set is a $\lambda$-filtered colimit of its subsets of cardinality smaller than $\lambda$ (partially ordered by inclusion).

An object $A$ of a category $\mathcal{C}$ is $\lambda$-presentable if the functor $\mathcal{C}(A,-)$ preserves $\lambda$-filtered colimits; that is, for each $\lambda$-filtered diagram $D: \mathcal{K} \rightarrow \mathcal{C}$ with a colimit $L$, each morphism $A \rightarrow L$ factors through a morphism $A \rightarrow D k$ for some $k \in \mathcal{K}$, and if two morphisms $A \rightarrow D k$ and $A \rightarrow D k^{\prime}$ compose to the same morphism $A \rightarrow L$, then there is some $k^{\prime \prime} \in \mathcal{K}$ and morphisms $k \rightarrow k^{\prime \prime}$ and $k^{\prime} \rightarrow k^{\prime \prime}$ in $\mathcal{K}$ such that the two composites $A \rightarrow D k^{\prime \prime}$ are equal (see $[26, \S 6.1]$ or $\left.[38, \S 2.1]\right)$.

For a small full subcategory $\mathcal{A}$ of $\mathcal{C}$ and an object $X$ in $\mathcal{C}$, the canonical diagram $(\mathcal{A} \downarrow X) \rightarrow \mathcal{C}$ sends each pair $\langle A, f\rangle$ with $f \in \mathcal{C}(A, X)$ to $A$. Recall from [2, 1.23] that $\mathcal{A}$ is called dense in $\mathcal{C}$ if each object $X$ of $\mathcal{C}$ is a colimit of the canonical diagram $(\mathcal{A} \downarrow X) \rightarrow \mathcal{C}$. A category $\mathcal{C}$ is bounded if it has a dense small full subcategory.

A category $\mathcal{C}$ is called $\lambda$-accessible if $\lambda$-filtered colimits exist in $\mathcal{C}$ and there is a set $\mathcal{A}$ of $\lambda$-presentable objects such that every object of $\mathcal{C}$ is a $\lambda$-filtered colimit of objects from $\mathcal{A}$. A category $\mathcal{C}$ is called accessible if it is $\lambda$-accessible for some regular cardinal $\lambda$. As shown in [2, p. 73] or [3, p. 226], if $\mathcal{C}$ is $\lambda$-accessible, then the full subcategory of its $\lambda$-presentable objects is essentially small and, if we denote by $\mathcal{C}_{\lambda}$ a set of representatives of all isomorphism classes of $\lambda$-presentable objects of $\mathcal{C}$, then $\mathcal{C}_{\lambda}$ is dense in $\mathcal{C}$. Moreover, for every $X \in \mathcal{C}$, the slice category $\left(\mathcal{C}_{\lambda} \downarrow X\right)$ is $\lambda$-filtered and $X$ is a colimit of the canonical diagram $\left(\mathcal{C}_{\lambda} \downarrow X\right) \rightarrow \mathcal{C}$. Thus, every accessible category is bounded.

An accessible category is called locally presentable if all colimits exist in it. It then follows, by [2, Corollary 1.28], that all limits exist as well. Every category of structures $\operatorname{Str} \Sigma$ is locally presentable [2, 5.1(5)], and the forgetful functor $\operatorname{Str} \Sigma \rightarrow$ Set $^{S}$ creates limits and colimits, where $S$ is the set of sorts of $\Sigma$ and $\operatorname{Set}^{S}$ denotes the category of $S$-sorted sets.

Theorem 7.1. Let $\lambda$ be a regular cardinal and let $\mathcal{C}$ be a $\lambda$-accessible category. Then there is a full embedding of $\mathcal{C}$ into a category of relational structures that preserves $\lambda$-filtered colimits.

Proof. Let us assume, with greater generality, that $\mathcal{C}$ is a bounded category and let $\mathcal{A}$ be a dense small full subcategory of $\mathcal{C}$. Denote by $\operatorname{Set}^{\mathcal{A}^{\text {op }}}$ the category of functors $\mathcal{A}^{\text {op }} \rightarrow$ Set, where $\mathcal{A}^{\mathrm{op}}$ is the opposite of $\mathcal{A}$. Then there are full embeddings

$$
\mathcal{C} \rightarrow \operatorname{Set}^{\mathcal{A}^{\mathrm{op}}} \rightarrow \operatorname{Str} \Sigma,
$$


defined as follows [2, Ch. 1]: The embedding of $\mathcal{C}$ into Set $^{\mathcal{A}^{\text {op }}}$ is of Yoneda type, sending each object $X$ to the restriction of $\mathcal{C}(-, X)$ to $\mathcal{A}^{\mathrm{op}}$. The fact that it is full and faithful is proved in [2, Proposition 1.26]. The signature $\Sigma$ is chosen by picking the objects of $\mathcal{A}$ as sorts and the morphisms of $\mathcal{A}^{\text {op }}$ as relation symbols. The full embedding of $\operatorname{Set}^{\mathcal{A}^{\text {op }}}$ into Str $\Sigma$ sends each functor $F$ to the $\mathcal{A}$-sorted set $\{F A: A \in \mathcal{A}\}$ together with a relation $\{(x,(F f) x): x \in F A\} \subset F A \times F B$ for each morphism $f: B \rightarrow A$ in $\mathcal{A}$. Hence, (7.1) sends each object $X \in \mathcal{C}$ to

$$
\langle\{\mathcal{C}(A, X): A \in \mathcal{A}\}, \quad\{\{(\alpha, \alpha \circ f): \alpha \in \mathcal{C}(A, X)\}: f \in \mathcal{A}(B, A)\}\rangle .
$$

If $\mathcal{C}$ is $\lambda$-accessible and we let $\mathcal{A}$ be a set of representatives of all isomorphism classes of $\lambda$-presentable objects in $\mathcal{C}$, then (7.1) preserves $\lambda$-filtered colimits, since the first arrow preserves $\lambda$-filtered colimits by [2, Proposition 1.26], and the second arrow preserves all filtered colimits (see [2, Example 1.41]).

As in [2, Definition 2.35], we say that a subcategory $\mathcal{C}$ of a category $\mathcal{D}$ is accessibly embedded if $\mathcal{C}$ is full and closed under $\lambda$-filtered colimits in $\mathcal{D}$ for some regular cardinal $\lambda$. Hence, in particular, $\mathcal{C}$ is isomorphism-closed, that is, every object of $\mathcal{D}$ which is isomorphic to an object of $\mathcal{C}$ is in $\mathcal{C}$. Moreover, the inclusion $\mathcal{C} \hookrightarrow \mathcal{D}$ creates $\lambda$-filtered colimits. If $\mathcal{D}$ is accessible and $\mathcal{C}$ is accessibly embedded into $\mathcal{D}$, then $\mathcal{C}$ is itself accessible if and only if, for some regular cardinal $\lambda$, every $\lambda$-filtered colimit of split subobjects of objects of $\mathcal{C}$ is in $\mathcal{C}$ (see [2, Corollary 2.36] for details).

Vopěnka's principle implies that every full embedding between accessible categories is accessible. The same conclusion can be inferred from the existence of sufficiently large $C(n)$-extendible cardinals [8].

A theory $T$ in a $\lambda$-ary language is called basic if each of its sentences has the form

$$
\forall\left\{x_{i}: i \in I\right\}\left(\varphi\left(x_{i}\right)_{i \in I} \rightarrow \psi\left(x_{i}\right)_{i \in I}\right)
$$

where $\varphi$ and $\psi$ are disjunctions of positive-primitive formulas and $|I|<\lambda$. A formula is positive-primitive if it has the form $\exists\left\{y_{j}: j \in J\right\} \eta\left(\left(y_{j}\right)_{j \in J},\left(z_{k}\right)_{k \in K}\right)$, in which $\eta$ is a conjunction of atomic formulas and $|J|,|K|<\lambda$.

It follows from Theorem 7.1 that every accessible category is equivalent to an accessibly embedded subcategory of a category of relational structures, namely to the closure of the image of (7.1) under isomorphisms. Moreover, the following fundamental fact is proved in [2]:

Theorem 7.2. Every accessibly embedded accessible subcategory of a category of structures is a category of models for some basic theory, and for every basic theory $T$ in some language $\mathcal{L}_{\lambda}(\Sigma)$, the category $\operatorname{Mod} T$ is accessible and accessibly embedded into $\operatorname{Str} \Sigma$.

Proof. This is shown in [2, Theorems 4.17 and 5.35].

We shall use the following terminology in order to simplify statements:

Definition 7.3. An accessible category of structures is a full subcategory of $\operatorname{Str} \Sigma$ that is accessible and accessibly embedded, for some signature $\Sigma$. 
We saw in Proposition 3.3 that each category $\operatorname{Mod} T$ is $\boldsymbol{\Delta}_{2}$ with parameters $\{\lambda, \Sigma, T\}$. Hence, Theorem 7.2 implies that every accessible category of structures is at most $\boldsymbol{\Delta}_{2}$. In many cases the complexity will be lower; for example, if $\Sigma$ is finitary, then, according to Proposition 3.3, $\operatorname{Mod} T$ is $\boldsymbol{\Delta}_{1}$ with parameters $\{\Sigma, T\}$. This amends the statement of [9, Proposition 4.2].

Although, in the rest of the article, we shall restrict most of our discussion to accessible categories of structures, results involving only concepts that are invariant under equivalence of categories will remain true for arbitrary accessible categories, by Theorem 7.1.

A regular cardinal $\kappa$ is said to be sharply bigger than another regular cardinal $\lambda$ if $\kappa>\lambda$ and, for each set $X$ of cardinality less than $\kappa$, the set $\mathcal{P}_{\lambda}(X)$ has a cofinal subset of cardinality less than $\kappa$. This notion was introduced in [38, §2.3], where it was proved that $\kappa$ is sharply bigger than $\lambda$ if and only if every $\lambda$-accessible category is $\kappa$-accessible (see also [2, Theorem 2.11]).

If $\kappa$ has the property that $v^{<\lambda}<\kappa$ for all $v<\kappa$ (which was used in Theorem 5.1 above) and $\kappa>\lambda$, then $\kappa$ is sharply bigger than $\lambda$, since, for a set $X$ of cardinality $\nu$, the cardinality of $\mathcal{P}_{\lambda}(X)$ is precisely $v^{<\lambda}$. Therefore, if $\lambda \leq \mu$, then $\left(2^{\mu}\right)^{+}$is sharply bigger than $\lambda$. This was first observed in [38, Proposition 2.3.5] and shows that for every $\lambda$ there are arbitrarily large regular cardinals sharply bigger than $\lambda$. Moreover, if $\kappa$ is strongly inaccessible and $\kappa>\lambda$, then $\kappa$ is sharply bigger than $\lambda$.

In what follows, for an $S$-sorted signature $\Sigma$ and a $\Sigma$-structure $A$, the cardinality of $A$ designates the sum $\sum_{s \in S}\left|A_{S}\right|$ of the cardinalities of the components of its underlying $S$-sorted set.

Lemma 7.4. Let $\Sigma$ be a $\lambda$-ary signature for a regular cardinal $\lambda$, and let $\mathcal{C}$ be a full $\lambda$-accessible subcategory of $\operatorname{Str} \Sigma$ closed under $\lambda$-filtered colimits. Let $\kappa$ be a regular cardinal sharply bigger than $\lambda$ and bigger than the cardinalities of all $\lambda$-presentable objects in $\mathcal{C}$, and such that $\Sigma \in H(\kappa)$. Then an object $A \in \mathcal{C}$ is $\kappa$-presentable if and only if its cardinality is smaller than $\kappa$.

Proof. Let $S$ be the set of sorts of $\Sigma$; let $\Sigma_{\text {op }}$ be its set of operation symbols and $\Sigma_{\text {rel }}$ its set of relation symbols. Let $A$ be a $\Sigma$-structure, and suppose first that its cardinality $\sum_{s \in S}\left|A_{s}\right|$ is smaller than $\kappa$. Let $D: \mathcal{K} \rightarrow \mathcal{C}$ be a $\kappa$-filtered diagram with a colimit $L$. Then $D$ is also $\lambda$-filtered and therefore the inclusion of $\mathcal{C}$ into $\operatorname{Str} \Sigma$ preserves its colimit. Suppose a homomorphism $f: A \rightarrow L$ is given. Since every set $A_{s}$ has cardinality less than $\kappa$ and $D$ is $\kappa$-filtered, each function $f_{s}: A_{s} \rightarrow L_{s}$ factors through $D\left(k_{s}\right)$ for some $k_{s} \in \mathcal{K}$. Since $|S|<\kappa$, we infer that $f$ factors (as a function) through $D k$ for some $k \in \mathcal{K}$. Moreover, since the cardinality of the set of all $\alpha$-sequences $\left\langle a_{i}: i \in \alpha\right\rangle$ with $a_{i} \in A_{s_{i}}$ for all $i$ and with $\alpha<\lambda$ is less than $\kappa$, and the cardinalities of the sets $\Sigma_{\text {op }}$ and $\Sigma_{\text {rel }}$ are also smaller than $\kappa$, we can find a morphism $k \rightarrow l$ in $\mathcal{K}$ such that the composite $A \rightarrow D k \rightarrow D l$ is a homomorphism of $\Sigma$-structures. For the same reason, given two homomorphisms $A \rightarrow D k$ and $A \rightarrow D k^{\prime}$ which coincide in $L$, there is an object $k^{\prime \prime} \in \mathcal{K}$ and morphisms $k \rightarrow k^{\prime \prime}$ and $k^{\prime} \rightarrow k^{\prime \prime}$ such that the composites $A \rightarrow D k \rightarrow D k^{\prime \prime}$ and $A \rightarrow D k^{\prime} \rightarrow D k^{\prime \prime}$ are equal. Hence $A$ is $\kappa$-presentable. 
For the converse, by [38, Proposition 2.3.11], if $\kappa$ is sharply bigger than $\lambda$ then every $\kappa$-presentable object $A$ in $\mathcal{C}$ is a $\lambda$-filtered colimit of $\lambda$-presentable objects indexed by a category with less than $\kappa$ morphisms. Therefore, since each $\lambda$-presentable object has cardinality smaller than $\kappa$ and the colimit is created in $\mathbf{S e t}^{S}$, it follows that $A$ has cardinality smaller than $\kappa$.

The following is our main result in this section.

Theorem 7.5. Let $\mathcal{C}$ be an accessible category of structures and let $\mathcal{S}$ be a $\boldsymbol{\Sigma}_{n}$ full subcategory of $\mathcal{C}$, where $n \geq 1$. Suppose that there is a proper class of supercompact cardinals if $n=2$ or that there is a proper class of $C(n-2)$-extendible cardinals if $n \geq 3$. Then there is a dense small full subcategory $\mathcal{D} \subseteq \mathcal{S}$ and there are arbitrarily large regular cardinals $\kappa$ such that, for all $Y \in \mathcal{S}$, the category $(\mathcal{D} \downarrow Y)$ is $\kappa$-filtered and $Y$ is a colimit of the canonical diagrams $(\mathcal{D} \downarrow Y) \rightarrow \mathcal{S}$ and $(\mathcal{D} \downarrow Y) \rightarrow \mathcal{C}$.

Proof. Note first that, if $\mathcal{S}$ is essentially small, then the result trivially holds with $\mathcal{D}$ a full subcategory of $\mathcal{S}$ containing one representative of each isomorphism class of objects in $\mathcal{S}$, if $\kappa$ is chosen bigger than the cardinality of the set of objects of $\mathcal{D}$. Therefore we assume from now on that there is a proper class of nonisomorphic objects in $\mathcal{S}$.

Choose a $\Sigma_{n}$ formula defining $\mathcal{S}$ with a set $p$ of parameters. Suppose that $\mathcal{C}$ embeds accessibly into $\operatorname{Str} \Sigma$ for a signature $\Sigma$, and pick a regular cardinal $\lambda$ such that $\Sigma$ is $\lambda$-ary and $\mathcal{C}$ is $\lambda$-accessible and closed under $\lambda$-filtered colimits in $\operatorname{Str} \Sigma$. Let $\mathcal{C}_{\lambda}$ be a set of representatives of all isomorphism classes of $\lambda$-presentable objects in $\mathcal{C}$.

Now let $\alpha$ be any given ordinal. Choose a regular cardinal $\kappa$ bigger than $\alpha$ and $\lambda$, and large enough so that each object in $\mathcal{C}_{\lambda}$ is in $H(\kappa)$ and $\{p, \Sigma\} \in H(\kappa)$ as well. Moreover, if $n=1$ then pick $\kappa$ of the form $\left(2^{\mu}\right)^{+}$with $\mu \geq \lambda$; if $n=2$ then choose instead $\kappa$ supercompact, and if $n \geq 3$ then choose it $C(n-2)$-extendible. With any of these choices, $\kappa$ is sharply bigger than $\lambda$ and therefore $\mathcal{C}$ is $\kappa$-accessible.

Let $\mathcal{D}$ be a full subcategory of $\mathcal{S}$ containing one representative of each isomorphism class of objects in the set $\mathcal{S} \cap H(\kappa)$. Note that, since each object of $\mathcal{D}$ is in $H(\kappa)$, all objects of $\mathcal{D}$ are $\kappa$-presentable in $\mathcal{C}$, by Lemma 7.4.

Let $\mathcal{C}_{\kappa}$ be a set of representatives of all isomorphism classes of $\kappa$-presentable objects of $\mathcal{C}$, chosen so that $\mathcal{D} \subseteq \mathcal{C}_{\kappa}$ and all objects of $\mathcal{C}_{\kappa}$ are in $H(\kappa)$. The latter is possible since, if $A \in \mathcal{C}$ and $A$ is $\kappa$-presentable, then $A$ has cardinality smaller than $\kappa$ by Lemma 7.4 and therefore $A \cong A^{\prime}$ as $\Sigma$-structures for some $A^{\prime} \in H(\kappa)$. Since $\mathcal{C}$ is isomorphism-closed, $A^{\prime}$ is in $\mathcal{C}$ and we may pick $A^{\prime}$ as a member of $\mathcal{C}_{\kappa}$.

Let $Y$ be any object of $\mathcal{S}$. Since $\mathcal{C}$ is $\kappa$-accessible, we know that $Y$ is a colimit of the canonical diagram $\left(\mathcal{C}_{\kappa} \downarrow Y\right) \rightarrow \mathcal{C}$, which is $\kappa$-filtered, by [2, p. 73]. Therefore, if we prove that $(\mathcal{D} \downarrow Y)$ is cofinal in $\left(\mathcal{C}_{\kappa} \downarrow Y\right)$, it will then follow that $Y$ is a colimit of the canonical diagram $(\mathcal{D} \downarrow Y) \rightarrow \mathcal{C}$, and that $(\mathcal{D} \downarrow Y)$ is $\kappa$-filtered. Moreover, since $Y$ is in $\mathcal{S}$, we shall be able to conclude that $Y$ is also a colimit of the canonical diagram $(\mathcal{D} \downarrow Y) \rightarrow \mathcal{S}$, as we wanted to show.

Thus, towards proving that $(\mathcal{D} \downarrow Y)$ is cofinal in $\left(\mathcal{C}_{\kappa} \downarrow Y\right)$, let $A$ be any object of $\mathcal{C}_{\kappa}$ and let a morphism $g: A \rightarrow Y$ be given. If $n=1$, then, since $A \in H(\kappa)$, it follows from part (a) of Theorem 5.1 that there is an object $\langle X, f\rangle$ in $(A \downarrow \mathcal{S})$ with $X \in \mathcal{S} \cap H(\kappa)$, 
together with an elementary embedding $e: X \rightarrow Y$ of $\Sigma$-structures such that $e \circ f=g$. If $n>1$, then Theorem 5.2 if $n=2$ and Theorem 6.6 if $n \geq 3$ lead to the same conclusion (recall that $H(\kappa)=V_{\kappa}$ if $\kappa$ is strongly inaccessible). In each case, we replace, if necessary, $X$ by an isomorphic object within $\mathcal{S} \cap H(\kappa)$, so we may assume that $X \in \mathcal{D}$.

We therefore have a commutative triangle

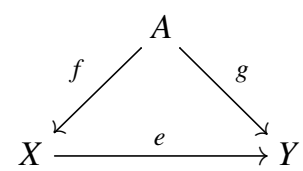

where $f$ can also be viewed as a morphism from $\langle A, g\rangle$ to $\langle X, e\rangle$ in $\left(\mathcal{C}_{\kappa} \downarrow Y\right)$. Since $\left(\mathcal{C}_{\kappa} \downarrow Y\right)$ is filtered, this tells us that $(\mathcal{D} \downarrow Y)$ is cofinal in $\left(\mathcal{C}_{\kappa} \downarrow Y\right)$, as we wanted to show.

Corollary 7.6. If there is a proper class of supercompact cardinals, then every accessible category is co-wellpowered.

Proof. Let $\mathcal{C}$ be an accessible category. Since accessibility and co-wellpoweredness are invariant under equivalence of categories, we can assume that $\mathcal{C}$ is a category of models of a basic theory $T$ for some signature $\Sigma$, by Theorems 7.1 and 7.2.

For an object $A \in \mathcal{C}$, let $\mathcal{E}_{A}$ be the full subcategory of $(A \downarrow \mathcal{C})$ whose objects are the epimorphisms. Then $\mathcal{E}_{A}$ is a partially ordered class, since between any two of its objects there is at most one morphism. Moreover, $\mathcal{E}_{A}$ is closed under colimits in $(A \downarrow \mathcal{C})$ and, if a diagram $D: \mathcal{K} \rightarrow \mathcal{E}_{A}$ has a colimit, then the colimit is a supremum of the set $\{D k: k \in \mathcal{K}\}$, hence determined by this set up to isomorphism. Therefore, in order to prove that $\mathcal{C}$ is co-wellpowered, it is enough to prove that $\mathcal{E}_{A}$ is bounded for every $A$, since this implies that $\mathcal{E}_{A}$ is essentially small.

From the fact that $\mathcal{C}$ is $\boldsymbol{\Delta}_{2}$ it follows that $\mathcal{E}_{A}$ is $\Pi_{2}$, since an object of $\mathcal{E}_{A}$ is a pair $\langle Y, g\rangle$ where $g \in \mathcal{C}(A, Y)$ and

$$
\forall Z \forall h \forall h^{\prime}\left[\left(h \in \mathcal{C}(Y, Z) \wedge h^{\prime} \in \mathcal{C}(Y, Z) \wedge h \circ g=h^{\prime} \circ g\right) \rightarrow h=h^{\prime}\right],
$$

and a morphism $\langle Y, g\rangle \rightarrow\left\langle Y^{\prime}, g^{\prime}\right\rangle$ is a morphism $d \in \mathcal{C}\left(Y, Y^{\prime}\right)$ with $g^{\prime}=d \circ g$. Hence, Theorem 7.5 implies that $\mathcal{E}_{A}$ is bounded under the assumption that there are arbitrarily large extendible cardinals.

However, as we next show, it is enough to assume that there are arbitrarily large supercompact cardinals. For this, we need to repeat the argument used in the proof of Theorem 7.5 and the one used in the proof of Theorem 5.2, adapted to our current situation.

If $\mathcal{C}$ is accessible, then $(A \downarrow \mathcal{C})$ is also accessible, by [2, Corollary 2.44]. Pick a regular cardinal $\lambda$ such that $(A \downarrow \mathcal{C})$ is $\lambda$-accessible. Assuming that there exists a proper class of supercompact cardinals, we may choose a supercompact cardinal $\kappa$ bigger than $\lambda$ such that $\Sigma, T \in H(\kappa)$ and such that all $\lambda$-presentable objects of $(A \downarrow \mathcal{C})$ are in $H(\kappa)$. Since $\kappa$ is strongly inaccessible, it is sharply bigger than $\lambda$ and therefore $(A \downarrow \mathcal{C})$ is $\kappa$-accessible.

Choose a full subcategory $\mathcal{D}$ of $\mathcal{E}_{A}$ containing one representative of each isomorphism class of objects in $\mathcal{E}_{A} \cap H(\kappa)$. By Lemma 7.4, all objects in $\mathcal{D}$ are $\kappa$-presentable. Choose 
also a set $(A \downarrow \mathcal{C})_{\kappa}$ of representatives of all isomorphism classes of $\kappa$-presentable objects of $(A \downarrow \mathcal{C})$, containing $\mathcal{D}$ and such that all its objects are in $H(\kappa)$, which is possible by Lemma 7.4.

Now let $\langle Y, g\rangle$ be any object of $\mathcal{E}_{A}$, so $g: A \rightarrow Y$ is an epimorphism. We know that $\langle Y, g\rangle$ is a colimit of the canonical diagram

$$
\left((A \downarrow \mathcal{C})_{\kappa} \downarrow\langle Y, g\rangle\right) \rightarrow(A \downarrow \mathcal{C}) .
$$

Hence it suffices to prove that $(\mathcal{D} \downarrow\langle Y, g\rangle)$ is cofinal in $\left((A \downarrow \mathcal{C})_{\kappa} \downarrow\langle Y, g\rangle\right)$. For this, pick any object in $\left((A \downarrow \mathcal{C})_{\kappa} \downarrow\langle Y, g\rangle\right)$, which consists of a $\kappa$-presentable object $\langle B, a\rangle$ of $(A \downarrow \mathcal{C})$ together with a morphism $d: B \rightarrow Y$ such that $d \circ a=g$. Pick a cardinal $\mu>\kappa$ such that $\langle Y, g\rangle \in H(\mu)$. Then $d$ is also in $H(\mu)$ since $B \in H(\kappa)$.

Let $j: V \rightarrow M$ be an elementary embedding with $M$ transitive and critical point $\kappa$, such that $j(\kappa)>\mu$ and $M$ is closed under $\mu$-sequences. Then $g$ and $d$ are in $M$ since $H(\mu) \in M$. Moreover, $\mathcal{C}$ is absolute between $M$ and $V$, by Proposition 3.3(b). Therefore $g$ is also an epimorphism in $M$, since, if $h, h^{\prime} \in \mathcal{C}(Y, Z)$ satisfy $h \circ g=h^{\prime} \circ g$ in $M$, then $h$ and $h^{\prime}$ also belong to $\mathcal{C}(Y, Z)$ in $V$ and therefore $h=h^{\prime}$, since $g$ is an epimorphism in $V$.

Since $Y \in H(\mu)$, the restriction $j\lceil Y: Y \rightarrow j(Y)$ is in $M$, and it is an elementary embedding of $\Sigma$-structures by Theorem 4.1. Since $A$ and $B$ are in $H(\kappa)$, we have that $j(A)=A$ and $j(B)=B$. Therefore, as in the proof of Theorem 5.2, $g: A \rightarrow Y$ and $d: B \rightarrow Y$ witness that in $M$ there is an object $X$ (namely, $Y$ ) and there is an epimorphism $f \in \mathcal{C}(A, X)$ with $\operatorname{rank}(X)<j(\kappa)$, together with an elementary embedding $e: X \rightarrow j(Y)$ such that $e \circ f=j(g)$ and a morphism $c \in \mathcal{C}(B, X)$ such that $c \circ a=f$ and $e \circ c=j(d)$. This implies, by elementarity of $j$, that in $V$ there is an epimorphism $f \in \mathcal{C}(A, X)$ with $\operatorname{rank}(X)<\kappa$, together with an elementary embedding $e: X \rightarrow Y$ such that $e \circ f=g$ and a morphism $c \in \mathcal{C}(B, X)$ such that $c \circ a=f$ and $e \circ c=d$. In other words, there is a commutative diagram

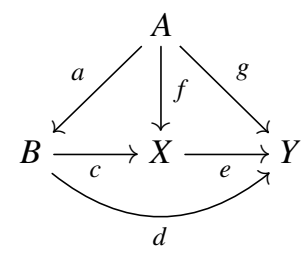

Here we may replace $\langle X, f\rangle$ by an isomorphic object which is in $\mathcal{D}$. This shows that $(\mathcal{D} \downarrow\langle Y, g\rangle)$ is cofinal in $\left((A \downarrow \mathcal{C})_{\kappa} \downarrow\langle Y, g\rangle\right)$, and consequently the category $\mathcal{E}_{A}$ is bounded, as needed.

On the other hand, as shown in [2, A.19], if each accessible category is co-wellpowered then there exists a proper class of measurable cardinals. Therefore, the statement that every accessible category is co-wellpowered is set-theoretical. Its precise consistency strength is not known (see [2, Open Problem 11]). By [38, Theorem 6.3.8(i)], together with the fact that categories of epimorphisms can be sketched by a pushout sketch (as done in [2, p.101]), the statement that every accessible category is co-wellpowered is 
implied by the existence of a proper class of strongly compact cardinals, a large-cardinal assumption that is not known to be weaker, consistency-wise, than the existence of a proper class of supercompact cardinals.

In order to simplify the statements of several corollaries of Theorem 7.5, we shall use the following terminology.

Definition 7.7. We say that a class $\mathcal{S}$ is definable with sufficiently low complexity if any of the following conditions is satisfied:

(1) $\mathcal{S}$ is $\boldsymbol{\Sigma}_{1}$.

(2) There is a proper class of supercompact cardinals and $\mathcal{S}$ is $\boldsymbol{\Sigma}_{2}$.

(3) There is a proper class of $C(n)$-extendible cardinals for some $n \geq 1$ and $\mathcal{S}$ is $\boldsymbol{\Sigma}_{n+2}$.

By Corollary 6.9, if Vopěnka's principle holds, then all classes are definable with sufficiently low complexity.

\section{Small-orthogonality classes}

An object $X$ and a morphism $f: A \rightarrow B$ in a category $\mathcal{C}$ are called orthogonal [25] if the function

$$
\mathcal{C}(f, X): \mathcal{C}(B, X) \rightarrow \mathcal{C}(A, X)
$$

is bijective. That is, $X$ and $f$ are orthogonal if and only if for every morphism $g: A \rightarrow X$ there is a unique morphism $h: B \rightarrow X$ such that $h \circ f=g$.

For a class of objects $\mathcal{X}$, we denote by ${ }^{\perp} \mathcal{X}$ the class of morphisms that are orthogonal to all the objects of $\mathcal{X}$. Similarly, for a class of morphisms $\mathcal{F}$, we denote by $\mathcal{F}^{\perp}$ the class of objects that are orthogonal to all the morphisms of $\mathcal{F}$. Classes of objects of the form $\mathcal{F}^{\perp}$ are called orthogonality classes, and, if $\mathcal{F}$ is a set (not a proper class), then $\mathcal{F}^{\perp}$ is a small-orthogonality class.

In what follows, we view each class of morphisms in $\mathcal{C}$ as a full subcategory of the category of arrows $\operatorname{Arr} \mathcal{C}$.

Lemma 8.1. For a regular cardinal $\lambda$, let $\mathcal{F}$ be a class of morphisms in a $\lambda$-accessible category $\mathcal{C}$, and let $\mathcal{D} \subseteq \mathcal{F}$. Suppose that each $f \in \mathcal{F}$ is a $\lambda$-filtered colimit of elements of $\mathcal{D}$ and suppose that the inclusion of $\mathcal{F}$ into $\operatorname{Arr} \mathcal{C}$ preserves the colimit. Then $\mathcal{D}^{\perp}=\mathcal{F}^{\perp}$.

Proof. To prove this, only the inclusion $\mathcal{D}^{\perp} \subseteq \mathcal{F}^{\perp}$ needs to be checked. Let $X \in \mathcal{D}^{\perp}$ and let $\langle A, B, f\rangle$ be any object of $\mathcal{F}$. By assumption, $\langle A, B, f\rangle \cong \operatorname{colim}\left\langle A_{k}, B_{k}, d_{k}\right\rangle$, where $\left\langle A_{k}, B_{k}, d_{k}\right\rangle$ is in $\mathcal{D}$ for all $k \in \mathcal{K}$, and $\mathcal{K}$ is $\lambda$-filtered. Since $\mathcal{C}$ is $\lambda$-accessible, the colimits colim $A_{k}$ and colim $B_{k}$ exist, and the induced arrow colim $A_{k} \rightarrow$ colim $B_{k}$ is a colimit of the arrows $d_{k}: A_{k} \rightarrow B_{k}$. Thus, $\operatorname{colim}\left\langle A_{k}, B_{k}, d_{k}\right\rangle \cong\left\langle\operatorname{colim} A_{k}, \operatorname{colim} B_{k}, \operatorname{colim} d_{k}\right\rangle$, so $f$ induces bijections

$$
\begin{aligned}
\mathcal{C}(B, X) & \cong \mathcal{C}\left(\operatorname{colim} B_{k}, X\right) \cong \lim \mathcal{C}\left(B_{k}, X\right) \\
& \cong \lim \mathcal{C}\left(A_{k}, X\right) \cong \mathcal{C}\left(\operatorname{colim} A_{k}, X\right) \cong \mathcal{C}(A, X),
\end{aligned}
$$

which means that $X \in \mathcal{F}^{\perp}$, as needed. 
Lemma 8.2. If $\mathcal{S}$ is a $\boldsymbol{\Sigma}_{n+1}$ full subcategory of a $\boldsymbol{\Sigma}_{n}$ category $\mathcal{C}$, then ${ }^{\perp} \mathcal{S}$ is $\boldsymbol{\Pi}_{n+1}$ if $n \geq 1$, and it is $\Pi_{2}$ if $n=0$.

Proof. The class ${ }^{\perp} \mathcal{S}$ can be defined as follows: $\langle A, B, f\rangle \in{ }^{\perp} \mathcal{S}$ if and only if

$$
\begin{aligned}
\forall X \forall g[(X \in \mathcal{S} \wedge g \in \mathcal{C}(A, X)) \rightarrow \exists h(h \in \mathcal{C}(B, X) \wedge h \circ f=g)] \\
\wedge \forall X \forall h_{1} \forall h_{2}\left[\left(X \in \mathcal{S} \wedge h_{1} \in \mathcal{C}(B, X) \wedge h_{2} \in \mathcal{C}(B, X)\right.\right. \\
\left.\left.\wedge h_{1} \circ f=h_{2} \circ f\right) \rightarrow h_{1}=h_{2}\right] .
\end{aligned}
$$

Recall that $P \rightarrow Q$ means $\neg(P \wedge \neg Q)$, or $\neg P \vee Q$. Therefore, (8.1) is at least $\Pi_{2}$, and it is $\Pi_{n+1}$ if $\mathcal{S}$ is $\boldsymbol{\Sigma}_{n+1}$ and $\mathcal{C}$ is at most $\boldsymbol{\Sigma}_{n}$ with $n \geq 1$.

Theorem 8.3. Assume the existence of a proper class of $C(n)$-extendible cardinals, where $n \geq 2$. Then each $\boldsymbol{\Sigma}_{n+1}$ orthogonality class in an accessible category $\mathcal{C}$ of structures is a small-orthogonality class.

Proof. Let $\mathcal{S}$ be a full subcategory of $\mathcal{C}$ whose objects form a $\boldsymbol{\Sigma}_{n+1}$ orthogonality class. Thus $\mathcal{S}=\mathcal{F}^{\perp}$ for some $\mathcal{F}$, and this implies that

$$
\left({ }^{\perp} \mathcal{S}\right)^{\perp}=\left({ }^{\perp}\left(\mathcal{F}^{\perp}\right)\right)^{\perp}=\mathcal{F}^{\perp}=\mathcal{S} .
$$

Since $\mathcal{C}$ is $\boldsymbol{\Delta}_{2}$ by Proposition 3.3, we infer from Lemma 8.2 that ${ }^{\perp} \mathcal{S}$ is $\boldsymbol{\Pi}_{n+1}$. Now the category of arrows $\operatorname{Arr} \mathcal{C}$ is accessible and embeds accessibly into a category of structures in such a way that complexity is preserved, by Lemma 3.2. Hence, by Theorem 7.5, ${ }^{\perp} \mathcal{S}$ has a dense small full subcategory $\mathcal{D}$ and there is a regular cardinal $\kappa$ (which we may choose so that $\mathcal{C}$ is $\kappa$-accessible) such that every arrow $f \in{ }^{\perp} \mathcal{S}$ is a $\kappa$-filtered colimit of elements of $\mathcal{D}$, both in ${ }^{\perp} \mathcal{S}$ and in $\operatorname{Arr} \mathcal{C}$. Then $\mathcal{D}^{\perp}=\left({ }^{\perp} \mathcal{S}\right)^{\perp}=\mathcal{S}$ by Lemma 8.1, so $\mathcal{S}$ is indeed a small-orthogonality class.

This result can be sharpened as follows. A reflection on a category is a left adjoint (when it exists) of the inclusion of a full subcategory [36], which is then called reflective. For example, in the category of groups, the abelianization functor is a reflection onto the reflective full subcategory of abelian groups. For every reflection $L$, the closure under isomorphisms of its image is an orthogonality class, and it is in fact orthogonal to the class of $L$-equivalences, i.e., morphisms $f$ such that $L f$ is an isomorphism.

A reflection $L$ is called an $\mathcal{F}$-reflection, where $\mathcal{F}$ is a set or a proper class of morphisms, if the closure under isomorphisms of the image of $L$ is equal to $\mathcal{F}^{\perp}$. This notion is particularly relevant when $\mathcal{F}$ can be chosen to be a set (or even better a single morphism). In the previous example, abelianization is an $f$-reflection where $f$ is the canonical projection of a free group on two generators onto a free abelian group on two generators, since the groups orthogonal to $f$ are precisely the abelian groups.

Theorem 8.4. Let $L$ be a reflection on an accessible category $\mathcal{C}$ of structures. Then $L$ is an $\mathcal{F}$-reflection for some set $\mathcal{F}$ of morphisms under any of the following assumptions:

(1) The class of L-equivalences is definable with sufficiently low complexity.

(2) The class of objects isomorphic to LX for some $X$ is $\boldsymbol{\Sigma}_{n+1}$ for $n \geq 2$ and there is a proper class of $C(n)$-extendible cardinals. 
Proof. To prove case (1), let $\mathcal{S}$ be the full subcategory of $L$-equivalences in the category of arrows of $\mathcal{C}$. It then follows from Theorem 7.5 that there is a small full subcategory $\mathcal{D}$ of $\mathcal{S}$ which is dense and satisfies $\mathcal{S}^{\perp}=\mathcal{D}^{\perp}$, by Lemma 8.1, as needed. Case (2) follows as a special case of Theorem 8.3.

The following corollary is a stronger variant of [9, Corollary 4.6]. The assumptions that $L$ be an epireflection and that $\mathcal{C}$ be balanced, which were made in [9], are not at all necessary here.

Corollary 8.5. Suppose that there is a proper class of supercompact cardinals. If $L$ is a reflection on an accessible category $\mathcal{C}$ of structures and the class of L-equivalences is $\boldsymbol{\Sigma}_{2}$, then $L$ is an $\mathcal{F}$-reflection for some set $\mathcal{F}$ of morphisms.

Proof. By assumption, the class of $L$-equivalences is definable with sufficiently low complexity. Hence, Theorem 8.4 applies.

As already shown in [17, Theorem 6.3], the assertion that every reflection on an accessible category is an $\mathcal{F}$-reflection for some set $\mathcal{F}$ of morphisms cannot be proved in ZFC. Specifically, if one assumes that measurable cardinals do not exist and considers reflection on the category of groups with respect to the class $\mathcal{Z}$ of homomorphisms of the form $\mathbb{Z}^{\kappa} / \mathbb{Z}^{<\kappa} \rightarrow\{0\}$, where $\kappa$ runs over all cardinals (see Example 2.2), then there is no set $\mathcal{F}$ of group homomorphisms such that $\mathcal{F}$-reflection coincides with $\mathcal{Z}$-reflection. This fact was also used in [9].

Theorem 8.6. If $\mathcal{C}$ is a locally presentable category of structures, then every full subcategory $\mathcal{S}$ of $\mathcal{C}$ closed under limits and definable with sufficiently low complexity is reflective.

Proof. As in the proof of Theorem 7.5, for every $A \in \mathcal{C}$ we can choose a small full subcategory $\mathcal{D}$ of $\mathcal{S}$ (depending on the cardinality of $A$ and the parameters of $\mathcal{C}$ ) such that every arrow $f: A \rightarrow Y$ with $Y$ in $\mathcal{S}$ factors through some object $X \in \mathcal{D}$. Hence the inclusion functor $\mathcal{S} \hookrightarrow \mathcal{C}$ satisfies the solution-set condition for every $A$ in $\mathcal{C}$, as required in the Freyd Adjoint Functor Theorem [36, V.6], from which the existence of a reflection of $\mathcal{C}$ onto $\mathcal{S}$ follows.

The following result is a further improvement, since it implies, among other things, that, if $\mathcal{S}$ is $\Sigma_{1}$, then the reflectivity of $\mathcal{S}^{\perp}$ is provable in ZFC. This yields, in particular, a solution of the Freyd-Kelly orthogonal subcategory problem [25] in ZFC for $\boldsymbol{\Sigma}_{1}$ classes.

Theorem 8.7. Let $\mathcal{S}$ be a class of morphisms definable with sufficiently low complexity in an accessible category $\mathcal{C}$ of structures. Then $\mathcal{S}^{\perp}$ is a small-orthogonality class and, if $\mathcal{C}$ is cocomplete, then $\mathcal{S}^{\perp}$ is reflective.

Proof. If we view $\mathcal{S}$ as a full subcategory of the category of arrows of $\mathcal{C}$, then Theorem 7.5 ensures that $\mathcal{S}$ has a dense small full subcategory $\mathcal{D}$ and Lemma 8.1 implies that $\mathcal{D}^{\perp}=\mathcal{S}^{\perp}$. Hence $\mathcal{S}^{\perp}$ is a small-orthogonality class, and small-orthogonality classes are reflective if colimits exist [2, 1.37].

If we weaken the assumption that $\mathcal{S}$ is closed under limits in Theorem 8.6, by imposing only that it is closed under products and retracts, then we may infer similarly that $\mathcal{S}$ 
is weakly reflective, under the hypotheses made in the statement. On the other hand, it is shown in [16] that, assuming the nonexistence of measurable cardinals, there is a $\Sigma_{2}$ full subcategory $\mathcal{S}$ of the category of abelian groups which is closed under products and retracts but not weakly reflective. Specifically, $\mathcal{S}$ is the closure of the class of groups $\mathbb{Z}^{\kappa} / \mathbb{Z}^{<\kappa}$ under products and retracts, where $\kappa$ runs over all cardinals. Hence, the statement that all $\boldsymbol{\Sigma}_{2}$ full subcategories closed under products and retracts in locally presentable categories are weakly reflective implies the existence of measurable cardinals, while it follows from the existence of supercompact cardinals.

Theorem 8.8. Every full subcategory closed under colimits and definable with sufficiently low complexity in a locally presentable category $\mathcal{C}$ of structures is coreflective.

Proof. Argue as in [2, Theorem 6.28].

\section{Consequences in homotopy theory}

Hovey conjectured in [29] that for every cohomology theory defined on spectra there is a homology theory with the same acyclics. This conjecture remains so far unsolved. In a different but closely related direction, the existence of cohomological localizations is also an open problem in ZFC, although it is known that it follows from Vopěnka's principle, both in unstable homotopy and in stable homotopy, by [17] and [15, Theorem 1.5].

Motivated by these problems, in this section we compare homological acyclic classes with cohomological acyclic classes from the point of view of complexity of their definitions. We consider homology theories and cohomology theories defined on simplicial sets and represented by spectra.

Spectra will be meant in the sense of Bousfield-Friedlander [13]. Thus, a spectrum E is a sequence of pointed simplicial sets

$$
\left\langle\left(E_{n}, p_{n}\right): p_{n} \in\left(E_{n}\right)_{0}, 0 \leq n<\omega\right\rangle
$$

equipped with pointed simplicial maps $\sigma_{n}: S E_{n} \rightarrow E_{n+1}$ for all $n$. Here $S$ denotes suspension, that is, $S X=\mathbb{S}^{1} \wedge X$. For $k \geq 1$, we denote by $\mathbb{S}^{k}$ the simplicial $k$-sphere, namely $\mathbb{S}^{k}=\Delta[k] / \partial \Delta[k]$, where $\Delta[k]$ is the standard $k$-simplex and $\partial \Delta[k]$ is its boundary. For pointed simplicial sets $X$ and $Y$, the smash product $X \wedge Y$ is the quotient of the product $X \times Y$ by the wedge sum $X \vee Y$, and we denote by $\operatorname{map}_{*}(X, Y)$ the pointed function complex from $X$ to $Y$, whose $n$-simplices are the pointed maps $X \wedge \Delta[n]_{+} \rightarrow Y$, where the subscript + means that a disjoint basepoint has been added.

A simplicial set is fibrant if it is a Kan complex [32]. For the purposes of this article, it will be convenient to use Kan's $\mathrm{Ex}^{\infty}$ construction as a fibrant replacement functor. Thus, there is a natural (injective) weak equivalence $j_{Y}: Y \hookrightarrow \mathrm{Ex}^{\infty} Y$ for all $Y$, where $\mathrm{Ex}^{\infty} Y$ is fibrant.

Let $[X, Y]$ denote the set of morphisms from $X$ to $Y$ in the pointed homotopy category of simplicial sets, which can be described as the set of pointed homotopy classes of maps $X \rightarrow \mathrm{Ex}^{\infty} Y$. If $Y$ is fibrant, then this is in bijective correspondence, via $j_{Y}$, with the set of pointed homotopy classes of maps $X \rightarrow Y$. 
A spectrum $E$ is an $\Omega$-spectrum if each $E_{n}$ is fibrant and the adjoints $\tau_{n}: E_{n} \rightarrow$ $\Omega E_{n+1}$ of the structure maps $\sigma_{n}: S E_{n} \rightarrow E_{n+1}$ are weak equivalences, where $\Omega$ denotes the loop space functor $\Omega X=\operatorname{map}_{*}\left(\mathbb{S}^{1}, X\right)$.

Each spectrum $E$ defines a reduced homology theory $E_{*}$ on simplicial sets by

$$
E_{k}(X)=\operatorname{colim}_{n} \pi_{n+k}\left(X \wedge E_{n}\right)=\operatorname{colim}_{n}\left[\mathbb{S}^{n+k}, X \wedge E_{n}\right]
$$

for $k \in \mathbb{Z}$, and, if $E$ is an $\Omega$-spectrum, then $E$ defines a reduced cohomology theory $E^{*}$ on simplicial sets by

$$
E^{k}(X)=\operatorname{colim}_{n} \pi_{n-k}\left(\operatorname{map}_{*}\left(X, E_{n}\right)\right)=\operatorname{colim}_{n}\left[S^{n} X, E_{n+k}\right]
$$

for $k \in \mathbb{Z}$. Note that, if $k \geq 0$, then simply $E^{k}(X) \cong\left[X, E_{k}\right]$.

Such homology or cohomology theories are called representable, and we shall only consider these in this article. Although not every generalized homology or cohomology theory in the sense of Eilenberg-Steenrod is representable [44, Example II.3.17], homological localizations have only been constructed and studied assuming representability [5], [11]. According to Brown's representability theorem, every cohomology theory which is additive (i.e., sending coproducts to products) is represented by some $\Omega$-spectrum. Similarly, homology theories that preserve filtered colimits are representable (see [4] or [44] for further details).

In most of what follows, we assume that $E$ is an $\Omega$-spectrum. A simplicial set $X$ is called $E_{*}$-acyclic if $E_{k}(X)=0$ for all $k \in \mathbb{Z}$, and, similarly, $X$ is $E^{*}$-acyclic if $E^{k}(X)=0$ for all $k \in \mathbb{Z}$. Observe that, by (9.2), the statement that $X$ is $E^{*}$-acyclic is equivalent to the statement that the pointed function complex $\operatorname{map}_{*}\left(X, E_{n}\right)$ is weakly contractible (that is, connected and with vanishing homotopy groups) for all $n$.

A map $f: X \rightarrow Y$ is an $E_{*}$-equivalence if $E_{k}(f): E_{k}(X) \rightarrow E_{k}(Y)$ is an isomorphism of abelian groups for all $k \in \mathbb{Z}$, and similarly for cohomology. Let $C f$ denote the mapping cone of $f$, which is obtained from the disjoint union of $Y$ and $X \times \Delta[1]$ by identifying $X \times\{0\}$ with $f(X) \subseteq Y$ using $f$, and collapsing $X \times\{1\}$ to a point. Using the Mayer-Vietoris axiom, one finds that $f$ is an $E_{*}$-equivalence if and only if $C f$ is $E_{*}$-acyclic, and analogously for cohomology.

The category of simplicial sets is $\Delta_{0}$, locally presentable, and it has a canonical accessible embedding into a category of structures with a finitary $\omega$-sorted operational signature. In fact, one can write down explicitly a formula without unbounded quantifiers expressing that $X$ and $Y$ are simplicial sets and $f$ is a simplicial map from $X$ to $Y$. This amounts to formalizing the claim that a simplicial set $X$ is a sequence of sets $\left\langle X_{n}: 0 \leq n<\omega\right\rangle$ (where the elements of $X_{n}$ are called $n$-simplices), together with functions $d_{i}^{n}: X_{n} \rightarrow X_{n-1}$ (called faces) for $n \geq 1$ and $0 \leq i \leq n$, and $s_{i}^{n}: X_{n} \rightarrow X_{n+1}$ (called degeneracies) for $n \geq 0$ and $0 \leq i \leq n$, satisfying the simplicial identities (see [40, Definition 1.1]). A simplicial map $f: X \rightarrow Y$ is a sequence of functions $\left\langle f_{n}: X_{n} \rightarrow Y_{n}\right\rangle_{0 \leq n<\omega}$ compatible with faces and degeneracies.

Similarly, the category of spectra is $\Delta_{0}$, locally presentable, and it also has an accessible embedding into a category of structures with a finitary $\omega$-sorted operational signature, since a spectrum $E$ is a sequence $\left\langle\left(E_{m}, p_{m}\right): 0 \leq m<\omega\right\rangle$ of pointed simplicial 
sets, where $p_{m} \in\left(E_{m}\right)_{0}$, plus a sequence $\left\langle\sigma_{m}: S E_{m} \rightarrow E_{m+1}\right\rangle_{0 \leq m<\omega}$ of pointed maps, each of which can be viewed as a map $\Delta[1] \times E_{m} \rightarrow E_{m+1}$ sending $\partial \Delta[1] \times E_{m}$ and $\Delta[1] \times\left\{p_{m}\right\}$ to the basepoint $p_{m+1}$. Giving a map $f: \Delta[1] \times E_{m} \rightarrow E_{m+1}$ is equivalent to giving a collection of functions

$$
f_{0}^{0}, f_{0}^{1}:\left(E_{m}\right)_{0} \rightarrow\left(E_{m+1}\right)_{0} \quad \text { and } \quad f_{k}^{0}, f_{k}^{1}, f_{k}^{01}:\left(E_{m}\right)_{k} \rightarrow\left(E_{m+1}\right)_{k}
$$

for $k \geq 1$, with commutativity conditions

$$
\begin{array}{lll}
f_{0}^{0} \circ d_{0}^{1}=d_{0}^{1} \circ f_{1}^{0}, & f_{0}^{1} \circ d_{0}^{1}=d_{0}^{1} \circ f_{1}^{1}, & f_{0}^{0} \circ d_{0}^{1}=d_{0}^{1} \circ f_{1}^{01}, \\
f_{0}^{0} \circ d_{1}^{1}=d_{1}^{1} \circ f_{1}^{0}, & f_{0}^{1} \circ d_{1}^{1}=d_{1}^{1} \circ f_{1}^{1}, & f_{0}^{1} \circ d_{1}^{1}=d_{1}^{1} \circ f_{1}^{01}, \\
s_{0}^{0} \circ f_{0}^{0}=f_{1}^{0} \circ s_{0}^{0}, & s_{0}^{0} \circ f_{0}^{1}=f_{1}^{1} \circ s_{0}^{0}, &
\end{array}
$$

and correspondingly for $k \geq 1$.

Proposition 9.1. The following are $\Delta_{1}$ classes:

(1) Fibrant simplicial sets.

(2) Weak equivalences of simplicial sets.

(3) Weakly contractible spectra.

(4) $\Omega$-spectra.

Proof. The assertion that a given simplicial set $X$ is fibrant can be formalized by means of the Kan extension condition, as in [40, Definition 1.3]. Explicitly, a simplicial set $X$ is fibrant if and only if for every $1 \leq n<\omega$ and every $k \leq n+1$, the following sentence holds: For all $x_{0}, x_{1}, \ldots, x_{n+1} \in X_{n}$ such that $d_{i}^{n} x_{j}=d_{j-1}^{n} x_{i}$ for $i<j, i \neq k$ and $j \neq k$, there exists $x \in X_{n+1}$ such that $d_{i}^{n+1} x=x_{i}$ for $i \neq k$. Since quantification over finite subsets is $\Delta_{1}$ (see Example 2.3), the class of fibrant simplicial sets is $\Delta_{1}$-definable.

Towards (2), recall that a map of simplicial sets $f: X \rightarrow Y$ is a weak equivalence if and only if it induces a bijection of connected components and isomorphisms of homotopy groups for every choice of a basepoint. Let us assume first that $X$ and $Y$ are fibrant. Then $f$ induces a bijection of connected components if and only if, for all $x_{0}$ and $x_{1}$ of $X_{0}$, if there exists $v \in Y_{1}$ with $d_{0}^{1} v=f\left(x_{0}\right)$ and $d_{1}^{1} v=f\left(x_{1}\right)$, then there exists $u \in X_{1}$ with $d_{0}^{1} u=x_{0}$ and $d_{1}^{1} u=x_{1}$, and moreover for each $y \in Y_{0}$ there exist $x \in X_{0}$ and $v \in Y_{1}$ such that $d_{0}^{1} v=y$ and $d_{1}^{1} v=f(x)$. Hence, the statement that $f$ induces a bijection of connected components is $\Delta_{0}$.

Similarly, if a simplicial set $X$ is fibrant, then the $n$th homotopy group $\pi_{n}(X, p)$ with basepoint $p \in X_{0}$ is the quotient of the set of all $x \in X_{n}$ such that $d_{i}^{n} x=s p$ for all $i$ (where $s=s_{n-2}^{n-2} \circ \cdots \circ s_{0}^{0}$ ) by the homotopy relation, where $x \sim x^{\prime}$ if $d_{i}^{n} x=d_{i}^{n} x^{\prime}$ for all $i$ and there exists $z \in X_{n+1}$ with $d_{n+1}^{n+1} z=x, d_{n}^{n+1} z=x^{\prime}$, and $d_{i}^{n+1} z=s_{n-1} d_{i}^{n} x$ for $0 \leq i<n$ (compare with [40, Definition 3.1]). Therefore, if $X$ and $Y$ are fibrant, then $f$ induces an isomorphism $\pi_{n}(X, p) \cong \pi_{n}(Y, q)$, where $p \in X_{0}$ and $q=f(p)$, if and only if the following sentence holds:

$$
\begin{aligned}
& \forall y \in Y_{n}\left[\forall i \leq n\left(d_{i}^{n} y=s q\right) \rightarrow\left[\exists x \in X _ { n } \left(\forall i \leq n\left(d_{i}^{n} x=s p\right)\right.\right.\right. \\
& \left.\left.\left.\quad \wedge f_{n}(x) \sim y \wedge \forall x^{\prime} \in X_{n}\left(\left(\forall i \leq n\left(d_{i}^{n} x^{\prime}=s p\right) \wedge f_{n}\left(x^{\prime}\right) \sim y\right) \rightarrow x \sim x^{\prime}\right)\right)\right]\right] .
\end{aligned}
$$


This shows that the statement that a map between fibrant simplicial sets is a weak equivalence is $\Delta_{1}$.

Next we analyze the complexity of a fibrant replacement. For a simplicial set $X$, the map $j_{X}: X \hookrightarrow \mathrm{Ex}^{\infty} X$ can be defined as the inclusion of $X$ into a simplicial set $\mathrm{Ex}^{\infty} X$ defined as follows. Let $\operatorname{Ex}^{1} X$ be the simplicial set whose set of $n$-simplices is the set of all maps from the barycentric subdivision of $\Delta[n]$ into $X$. The barycentric subdivision sd $\Delta[n]$ is the nerve of the poset of nondegenerate simplices of $\Delta[n]$ (see [27, Ch. III, $\S 4])$. The last vertex map $\mathrm{sd} \Delta[n] \rightarrow \Delta[n]$ yields an inclusion $X \hookrightarrow \operatorname{Ex}^{1} X$. Then $\operatorname{Ex}^{\infty} X$ is the union of a sequence of inclusions $\operatorname{Ex}^{k} X \hookrightarrow \mathrm{Ex}^{k+1} X$ for $k \geq 1$, where $\mathrm{Ex}^{k}$ is the composite of $\mathrm{Ex}^{1}$ with itself $k$ times.

Let $p$ be any vertex of $X$. Each element in $\pi_{n}\left(\mathrm{Ex}^{\infty} Y, f(p)\right)$ is represented by a map $\mathbb{S}^{n} \rightarrow \operatorname{Ex}^{k} Y$ based at $f(p)$ for some $k<\omega$; that is, a map from $\Delta[n]$ to $\operatorname{Ex}^{k} Y$ sending the boundary of $\Delta[n]$ to $f(p)$. By adjointness, the maps $\Delta[n] \rightarrow \operatorname{Ex}^{k} Y$ correspond bijectively to the maps $\operatorname{sd}^{k} \Delta[n] \rightarrow Y$, where $\mathrm{sd}^{k}$ is an iterated barycentric subdivision. Let $a_{k, n}$ be the number of nondegenerate $n$-simplices of $\operatorname{sd}^{k} \Delta[n]$ and let $R_{k, n}$ be the set of all relations among their faces. For example, $a_{2,1}=4$ and $R_{2,1}$ consists of the equalities

$$
d_{1}^{1} x_{(0 \rightarrow 001)}=d_{1}^{1} x_{(01 \rightarrow 001)}, \quad d_{0}^{1} x_{(01 \rightarrow 001)}=d_{0}^{1} x_{(01 \rightarrow 011)}, \quad d_{1}^{1} x_{(01 \rightarrow 011)}=d_{1}^{1} x_{(1 \rightarrow 011)} .
$$

Thus, each map $\Delta[n] \rightarrow \operatorname{Ex}^{k} Y$ is determined by a sequence of $a_{k, n}$ (not necessarily distinct) elements of $Y_{n}$ satisfying a set $R_{k, n}$ of equalities among their faces. In what follows, when we write "a map $\beta: \mathbb{S}^{n} \rightarrow \operatorname{Ex}^{k} Y$ " we implicitly formalize it as an ordered sequence of $a_{k, n}$ elements of $Y_{n}$ satisfying a set $S_{k, n}$ of sentences, including those of $R_{k, n}$ and those needed to express the fact that $\partial \Delta[n]$ is sent to the basepoint $f(p)$. Homotopies into $\mathrm{Ex}^{k} Y$ are formalized similarly.

The assertion that a map $f: X \rightarrow Y$ induces $\pi_{n}\left(\mathrm{Ex}^{\infty} X, p\right) \cong \pi_{n}\left(\mathrm{Ex}^{\infty} Y, f(p)\right)$ for every $p \in X_{0}$ can therefore be expressed by stating that for every $k<\omega$ and every map $\beta: \mathbb{S}^{n} \rightarrow \operatorname{Ex}^{k} Y$ based at $f(p)$ there exist $l<\omega$ and a map $\alpha: \mathbb{S}^{n} \rightarrow \operatorname{Ex}^{l} X$ based at $p$ and a homotopy $H: \mathbb{S}^{n} \wedge \Delta[1]_{+} \rightarrow \operatorname{Ex}^{r} Y$ from $\left(\operatorname{Ex}^{r} f\right) \circ \alpha$ to $\beta$, where $r \geq k$ and $r \geq l$, and, moreover, if $\alpha^{\prime}: \mathbb{S}^{n} \rightarrow \operatorname{Ex}^{m} X$ is based at $p$ and there is a homotopy from $\left(\operatorname{Ex}^{r} f\right) \circ \alpha^{\prime}$ to $\beta$ with $r \geq k$ and $r \geq m$, then there is a homotopy $H: \mathbb{S}^{n} \wedge \Delta[1]_{+} \rightarrow \operatorname{Ex}^{s} X$ from $\alpha$ to $\alpha^{\prime}$ with $s \geq l$ and $s \geq m$. Therefore, the class of weak equivalences between simplicial sets is $\Delta_{1}$-definable.

Having proved (1) and (2), we next address (3). A spectrum $F$ is weakly contractible if and only if all its homotopy groups vanish, that is,

$$
\operatorname{colim}_{n}\left[\mathbb{S}^{n+k}, F_{n}\right]=0 \quad \text { for all } k \in \mathbb{Z}
$$

This is equivalent to imposing that, for all $k \in \mathbb{Z}$ and $n \geq 0$ such that $n+k \geq 0$, each pointed map $\beta: \mathbb{S}^{n+k} \rightarrow \operatorname{Ex}^{\infty} F_{n}$ becomes nullhomotopic after suspending it a finite number of times (say, $m$ times) and composing with the structure maps $\sigma_{n}: S F_{n} \rightarrow F_{n+1}$. More precisely, on the one hand, we have

$$
\mathbb{S}^{n+m+k} \stackrel{S^{m} \beta}{\longrightarrow} S^{m} \mathrm{Ex}^{\infty} F_{n} \stackrel{j}{\rightarrow} \mathrm{Ex}^{\infty} S^{m} \mathrm{Ex}^{\infty} F_{n}
$$


and, on the other hand, there are maps

$$
\mathrm{Ex}^{\infty} S^{m} \mathrm{Ex}^{\infty} F_{n} \stackrel{\mathrm{Ex}^{\infty} S^{m} j}{\longleftarrow} \mathrm{Ex}^{\infty} S^{m} F_{n} \stackrel{\mathrm{Ex}^{\infty} \sigma}{\longrightarrow} \mathrm{Ex}^{\infty} F_{n+m}
$$

where $\sigma$ is an abbreviation for $\sigma_{n+m-1} \circ S \sigma_{n+m-2} \circ \cdots \circ S^{m-2} \sigma_{n+1} \circ S^{m-1} \sigma_{n}$. The maps $j$ and $\mathrm{Ex}^{\infty} S^{m} j$ are natural weak equivalences.

Hence, $F$ is weakly contractible if and only if, for each $k \in \mathbb{Z}$ and each $(n+k)$-simplex $x \in \mathrm{Ex}^{\infty} F_{n}$ whose faces are equal to the basepoint, there is an $(n+m+k)$-simplex $y \in \mathrm{Ex}^{\infty} S^{m} F_{n}$ whose faces are equal to the basepoint and an $(n+m+k+1)$-simplex $z \in \mathrm{Ex}^{\infty} F_{n+m}$ whose top face is $y$ and all its other faces are equal to the basepoint, and $\left(\mathrm{Ex}^{\infty} S^{m} j\right) y \sim j\left(S^{m} x\right)$.

We finally prove (4). In order to formalize the fact that a spectrum $E$ is an $\Omega$-spectrum, we first need that each simplicial set $E_{n}$ be fibrant. Then we need to define the adjoint maps $\tau_{n}: E_{n} \rightarrow \Omega E_{n+1}$ and we need to impose that each $\tau_{n}$ be a weak equivalence. To define $\tau_{n}$, let $x$ be a $k$-simplex of $E_{n}$. Its image in $\Omega E_{n+1}=\operatorname{map}_{*}\left(\mathbb{S}^{1}, E_{n+1}\right)$ is a map $\mathbb{S}^{1} \wedge \Delta[k]_{+} \rightarrow E_{n+1}$ which is determined by imposing that

$$
\left(\tau_{n}(x)\right)\left(s e_{1}, e_{k}\right)=\sigma_{n}\left(s e_{1}, x\right),
$$

where $e_{1}$ is the nondegenerate 1 -simplex of $\mathbb{S}^{1}$ and $e_{k}$ is the nondegenerate $k$-simplex of $\Delta[k]$, and $s$ denotes a composition of degeneracies.

In what follows, we denote by $\mathbf{s S e t}_{*}$ the category of pointed simplicial sets and pointed maps.

Theorem 9.2. The class of $E_{*}$-acyclic simplicial sets for a spectrum $E$ is $\boldsymbol{\Delta}_{1}$ with $E$ as a parameter.

Proof. If $(X, p)$ and $(Y, q)$ are pointed simplicial sets, then $W=X \vee Y$ is a pointed simplicial set contained in $X \times Y$ such that $W_{n}$ contains all elements of the form $(x, s q)$ with $x \in X_{n}$ and all those of the form $(s p, y)$ with $y \in Y_{n}$, where $s$ is a composition of degeneracies, with basepoint $(p, q)$. The smash product $X \wedge Y$ is obtained from $X \times Y$ by collapsing $X \vee Y$ to a point. Hence, $(X \wedge Y)_{n}=\left(X_{n} \times Y_{n}\right) \backslash\left(W_{n} \backslash\{(s p, s q)\}\right)$ for all $n$, and we declare equal to $(s p, s q)$ all faces of elements of $X_{n+1} \times Y_{n+1}$ and all degeneracies of elements of $X_{n-1} \times Y_{n-1}$ taking values in $W_{n}$.

If $(X, p)$ is a pointed simplicial set and $E$ is a spectrum with structure maps $\left\langle\sigma_{n}\right.$ : $0 \leq n<\omega\rangle$, then $X \wedge E$ is a spectrum with $(X \wedge E)_{n}=X \wedge E_{n}$ and structure maps (id $\left.\wedge \sigma_{n}\right) \circ\left(\tau \wedge\right.$ id) for all $n$, where $\tau: \mathbb{S}^{1} \wedge X \rightarrow X \wedge \mathbb{S}^{1}$ is the twist map. By Proposition 9.1(3), the statement that $X \wedge E$ is weakly contractible is $\Delta_{1}$. However, a formula expressing this fact has to contain a definition of $X \wedge E$, where $E$ is a given spectrum treated as a parameter. This can be done in two equivalent ways, as follows:

$$
\begin{aligned}
X \in \mathbf{S S e t}_{*} \wedge & \exists F\left[F \text { is a spectrum } \wedge ( \forall n < \omega ) \left(\left(F_{n}=X \wedge E_{n}\right)\right.\right. \\
& \left.\left.\wedge \sigma_{n}^{F}=\left(\mathrm{id} \wedge \sigma_{n}^{E}\right) \circ(\tau \wedge \text { id })\right) \wedge F \text { is weakly contractible }\right] ; \\
X \in \mathbf{S S e t}_{*} \wedge & \forall F\left[\left[F \text { is a spectrum } \wedge ( \forall n < \omega ) \left(\left(F_{n}=X \wedge E_{n}\right)\right.\right.\right. \\
& \left.\left.\left.\wedge \sigma_{n}^{F}=\left(\text { id } \wedge \sigma_{n}^{E}\right) \circ(\tau \wedge \text { id })\right)\right] \rightarrow F \text { is weakly contractible }\right] .
\end{aligned}
$$

Since (9.4) is $\Sigma_{1}$ and (9.5) is $\Pi_{1}$, the theorem is proved. 
As explained in Section 2, the fact that homological acyclic classes are $\boldsymbol{\Delta}_{1}$ implies that they are absolute. This means that, if $E$ is a spectrum and $M$ is a transitive model of ZFC such that $E \in M$ (in which case $E$ is a spectrum in $M$ as well, since being a spectrum is $\Delta_{0}$ ), then a simplicial set $X \in M$ is $E_{*}$-acyclic in $M$ if and only if it is $E_{*}$-acyclic.

We thank Federico Cantero for pertinent remarks about the argument given in the proof of the next result.

Theorem 9.3. The class of $E^{*}$-acyclic simplicial sets for an $\Omega$-spectrum $E$ is $\boldsymbol{\Delta}_{2}$ with $E$ as a parameter.

Proof. Let $E$ be an $\Omega$-spectrum, which will be used as a parameter. By Proposition 9.1(4), every transitive model of ZFC containing $E$ will agree with the fact that $E$ is an $\Omega$-spectrum.

A simplicial set $X$ is $E^{*}$-acyclic if and only if, for all $k \in \mathbb{Z}$ and $n \geq 0$ with $n+k \geq 0$, every map $S^{n} X \rightarrow E_{n+k}$ becomes nullhomotopic after suspending it a finite number of times and composing with the structure maps of $E$ as in (9.3). This claim leads to a $\Pi_{2}$ formula-note that a map $S^{n} X \rightarrow E_{n+k}$ is no longer determined by any finite set of simplices of $E_{n+k}$. Next we show that it is possible to restate it by means of a $\Sigma_{2}$ formula.

A pointed simplicial set $(X, p)$ is $E^{*}$-acyclic if and only if for all $n<\omega$ the simplicial set $\operatorname{map}_{*}\left(X, E_{n}\right)$ is weakly contractible, assuming that $E$ is an $\Omega$-spectrum. Thus, $X$ is $E^{*}$-acyclic if and only if the following sentence is true, where we need to include a definition of $M=\operatorname{map}_{*}\left(X, E_{n}\right)$ :

$$
\begin{aligned}
X \in \mathbf{S S e t}_{*} & \wedge(\forall n<\omega) \exists M\left[M \in \mathbf{S S e t}_{*}\right. \\
& \wedge(\forall k<\omega)\left[\left(\forall f \in M_{k}\right) f \in \mathbf{s S e t}_{*}\left(X \wedge \Delta[k]_{+}, E_{n}\right)\right. \\
& \left.\left.\wedge \forall g\left(g \in \mathbf{S S e t}_{*}\left(X \wedge \Delta[k]_{+}, E_{n}\right) \rightarrow g \in M_{k}\right)\right] \wedge M \text { is weakly contractible }\right] .
\end{aligned}
$$

According to Proposition 9.1, this is a $\Sigma_{2}$ formula.

In order to state and prove the next results, we use the term homotopy reflection (also called homotopy localization elsewhere) to designate a functor $L: \mathbf{s S e t}_{*} \rightarrow \mathbf{s S e t}_{*}$ equipped with a natural transformation $\eta$ : Id $\rightarrow L$ which preserves weak equivalences and becomes a reflection when passing to the homotopy category. For a homotopy reflection $L$, an $L$-equivalence is a map $f: X \rightarrow Y$ such that $L f: L X \rightarrow L Y$ is an isomorphism in the homotopy category, and a simplicial set $X$ is called L-local if it is fibrant and weakly equivalent to $L X$ for some $X$.

We also recall that, for a pointed map $f: A \rightarrow B$, a connected fibrant simplicial set $X$ is $f$-local if the induced map of pointed function complexes

$$
\operatorname{map}_{*}(f, X): \operatorname{map}_{*}(B, X) \rightarrow \operatorname{map}_{*}(A, X)
$$

is a weak equivalence, and a disconnected $X$ is $f$-local if each of its connected components is $f$-local with any choice of basepoint (cf. [21, 1.A.1]). Note that, if $X$ is $f$-local for a map $f: A \rightarrow B$, then $f$ induces a bijection $[B, X] \cong[A, X]$, since $[B, X]$ is in natural bijective correspondence with the set of connected components of $\operatorname{map}_{*}(B, X)$. 
Hence, being $f$-local is a stronger condition than being orthogonal to $f$ in the homotopy category.

The same terminology is used for a set or a proper class of maps $\mathcal{F}$, that is, a simplicial set is $\mathcal{F}$-local if it is $f$-local for all $f \in \mathcal{F}$. An $\mathcal{F}$-localization is a homotopy reflection $L$ such that the class of $L$-local spaces coincides with the class of $\mathcal{F}$-local spaces.

Lemma 9.4. Given any class $\mathcal{S}$ of pointed maps between simplicial sets, if there is a subclass $\mathcal{F} \subseteq \mathcal{S}$ such that each element of $\mathcal{S}$ is a filtered colimit of elements of $\mathcal{F}$, then every $\mathcal{F}$-local space is $\mathcal{S}$-local.

Proof. The argument is analogous to the one in the proof of Lemma 8.1. Let $f: A \rightarrow B$ be any element of $\mathcal{S}$ and let $X$ be an $\mathcal{F}$-local simplicial set, which we may assume connected. Write $f=$ colim $f_{k}$ (in the category of pointed maps between simplicial sets), where $f_{k}: A_{k} \rightarrow B_{k}$ is in $\mathcal{F}$ for all $k \in \mathcal{K}$, and $\mathcal{K}$ is filtered. Now we use, as in [17, Lemma 5.2], the fact that the natural map

$$
\operatorname{hocolim} f_{k} \rightarrow \operatorname{colim} f_{k}
$$

is a weak equivalence, since homotopy groups commute with filtered colimits (hocolim denotes a pointed homotopy colimit $[28,18.8])$. Hence,

$$
\begin{aligned}
\operatorname{map}_{*}(B, X) & \simeq \operatorname{map}_{*}\left(\operatorname{hocolim} B_{k}, X\right) \simeq \operatorname{holim}_{\operatorname{map}_{*}}\left(B_{k}, X\right) \\
& \simeq \operatorname{holim}_{*} \operatorname{map}_{*}\left(A_{k}, X\right) \simeq \operatorname{map}_{*}\left(\operatorname{hocolim} A_{k}, X\right) \simeq \operatorname{map}_{*}(A, X),
\end{aligned}
$$

from which it follows indeed that $X$ is $\mathcal{S}$-local.

Theorem 9.5. Assume the existence of arbitrarily large supercompact cardinals. Then for every additive cohomology theory $E^{*}$ defined on simplicial sets there is a homotopy reflection $L$ such that the $L$-equivalences are precisely the $E^{*}$-equivalences.

Proof. Let $\mathcal{S}$ be the class of $E^{*}$-equivalences for a given additive cohomology theory $E^{*}$, and view it as a full subcategory of the category of pointed maps between simplicial sets, which is accessibly embedded into a category of structures, by Lemma 3.2. Since the class of $E^{*}$-equivalences coincides with the class of maps whose mapping cone is $E^{*}$-acyclic, Theorem 9.3 tells us that $\mathcal{S}$ is $\boldsymbol{\Delta}_{2}$, hence $\boldsymbol{\Sigma}_{2}$. Consequently, it follows from Theorem 7.5 that there is a regular cardinal $\kappa$ and a set $\mathcal{F}$ of $E^{*}$-equivalences such that every $E^{*}$-equivalence is a $\kappa$-filtered colimit of elements of $\mathcal{F}$ in the category of pointed maps between simplicial sets.

To conclude the proof, let $f: A \rightarrow B$ be the coproduct of all the elements of $\mathcal{F}$, and let $L$ be $f$-localization, as constructed in [12], [21] or [28]. Since all the elements of $\mathcal{F}$ are $E^{*}$-equivalences and $E^{*}$ is additive, $f$ is an $E^{*}$-equivalence.

Let $E$ be an $\Omega$-spectrum representing $E^{*}$. Since $f$ is an $E^{*}$-equivalence, it induces bijections $\left[B, E_{n}\right] \cong\left[A, E_{n}\right]$ for all $n$, and in fact weak equivalences $\operatorname{map}_{*}\left(B, E_{n}\right) \simeq$ $\operatorname{map}_{*}\left(A, E_{n}\right)$ for all $n$. In other words, the basepoint component of $E_{n}$ is $f$-local for all $n$. Since $E_{n}$ is a loop space, all its connected components have the same homotopy type and therefore $E_{n}$ itself is $f$-local for all $n$. It follows that every $L$-equivalence $g: X \rightarrow Y$ 
induces a weak equivalence $\operatorname{map}_{*}\left(Y, E_{n}\right) \simeq \operatorname{map}_{*}\left(X, E_{n}\right)$ for all $n$, and we conclude that all $L$-equivalences are $E^{*}$-equivalences.

Conversely, every $E^{*}$-equivalence is, as said above, a $\kappa$-filtered colimit of objects from $\mathcal{F}$. According to Lemma 9.4, every $L$-local simplicial set is $E^{*}$-local, and therefore all $E^{*}$-equivalences are $L$-equivalences. This completes the argument.

What we have proved is that localization with respect to any additive cohomology theory exists on the homotopy category of simplicial sets if there exist arbitrarily large supercompact cardinals. This is a substantial improvement of [17, Corollary 5.4], where the existence of cohomological localizations was inferred from Vopěnka's principle.

We also emphasize that from Theorem 9.2 it follows, by a similar method to the proof of Theorem 9.5 (or using Theorem 9.7 below), that the existence of homological localizations (for representable homology theories) is provable in ZFC. Bousfield did it indeed in [11].

The same line of argument provides an answer to Farjoun's question in [20] of whether all homotopy reflections are $f$-localizations for some map $f$. It was shown in [17] that the answer is affirmative under Vopěnka's principle, and Przeździecki proved in [42] that an affirmative answer is in fact equivalent to Vopěnka's principle. Here we prove an analogue of Theorem 8.4.

Theorem 9.6. A homotopy reflection $L$ on simplicial sets is an $f$-localization for some map $f$ under any of the following assumptions:

(1) The class of L-equivalences is definable with sufficiently low complexity.

(2) The class of L-local simplicial sets is $\boldsymbol{\Sigma}_{n+1}$ for $n \geq 2$ and there is a proper class of $C(n)$-extendible cardinals.

Proof. For (1), we may choose, by Theorem 7.5, a set $\mathcal{F}$ of $L$-equivalences such that every $L$-equivalence is a filtered colimit of elements of $\mathcal{F}$ in the category of pointed maps between simplicial sets. Let $f$ be the coproduct of all the elements of $\mathcal{F}$. Then $f$ is an $L$-equivalence, since the class of $L$-equivalences is closed under coproducts. Therefore, every $L$-local simplicial set is $f$-local, by [17, Corollary 4.4]. Conversely, every $f$-local simplicial set is $L$-local by Lemma 9.4.

In order to prove (2), note that, if the class of $L$-local simplicial sets is $\boldsymbol{\Sigma}_{n+1}$, then the class of $L$-equivalences is $\Pi_{n+1}$, since $f: A \rightarrow B$ is an $L$-equivalence if and only if the induced function $[B, X] \rightarrow[A, X]$ is a bijection for each $L$-local space $X$, which can be formalized as

$\forall X \forall g\left[\left(X\right.\right.$ is an $L$-local simplicial set $\wedge g \in$ sSet $\left._{*}(A, X)\right) \rightarrow$

$\left(\exists h\left(h \in \operatorname{sSet}_{*}(B, X) \wedge h \circ f \simeq g\right) \wedge\right.$ any two such maps are homotopic)].

The statement "any two such maps are homotopic" can be formally written as a $\Pi_{2}$ formula. Hence the same argument as in part (1) applies under the assumption that a proper class of $C(n)$-extendible cardinals exists, by means of Theorem 7.5.

The corresponding analogue of Theorem 8.7 is the next result. Localization with respect to proper classes of maps was shown to exist in [18] under restrictive conditions. 
Theorem 9.7. Let $\mathcal{S}$ be any (possibly proper) class of maps of simplicial sets. If $\mathcal{S}$ is definable with sufficiently low complexity, then an $\mathcal{S}$-localization exists.

Proof. Theorem 7.5 implies that there is a set $\mathcal{F} \subseteq \mathcal{S}$ such that every $f \in \mathcal{S}$ is a filtered colimit of elements of $\mathcal{F}$. Then $\mathcal{F}$-localization exists since $\mathcal{F}$ is a set, and every $\mathcal{F}$-local simplicial set is $\mathcal{S}$-local by Lemma 9.4. Since $\mathcal{F} \subseteq \mathcal{S}$, all $\mathcal{S}$-local simplicial sets are $\mathcal{F}$-local, so the proof is complete.

\section{Bergman's question}

If $\Sigma$ is a finitary operational signature, then $\Sigma$-structures are universal algebras. If $\mathcal{C}$ is a full subcategory of $\operatorname{Str} \Sigma$ and $n$ is a nonnegative integer, an $n$-ary implicit operation $f$ on $\mathcal{C}$ is a collection of functions $f_{X}: X^{n} \rightarrow X$ indexed by objects $X$ of $\mathcal{C}$ such that the square

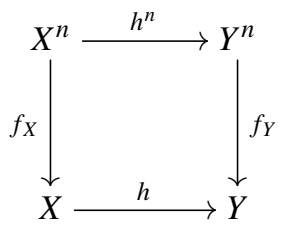

commutes for each homomorphism $h: X \rightarrow Y$. Such implicit operations are very useful in finite universal algebra (see [6]). If $\mathcal{C}$ is a proper class with no homomorphisms except identities, then each collection $\left\{f_{X}\right\}_{X \in \mathcal{C}}$ is an implicit operation. In a previous version of [10], Bergman asked if it can happen that there is a proper class of distinct implicit operations on some $\mathcal{C}$ assuming Vopěnka's principle (here we neglect the fact that implicit operations need not be sets themselves).

Theorem 10.1. For a finitary operational signature $\Sigma$, Vopěnka's principle implies that there is only a set of implicit operations on each full subcategory of $\operatorname{Str} \Sigma$.

Proof. Let $\mathcal{C}$ be a full subcategory of $\operatorname{Str} \Sigma$, where $\Sigma$ is $S$-sorted. By [3], Vopěnka's principle implies that there is a regular cardinal $\kappa$ and a set $\mathcal{A}$ of objects in $\mathcal{C}$ such that each object of $\mathcal{C}$ is a $\kappa$-filtered colimit of objects of $\mathcal{A}$. Now, since the forgetful functor $\operatorname{Str} \Sigma \rightarrow$ Set $^{S}$ and the $n$-fold product functor $(-)^{n}: \mathbf{S e t}^{S} \rightarrow \mathbf{S e t}^{S}$ preserve colimits, each implicit operation $f_{X}$ with $X \in \mathcal{C}$ is uniquely determined by $\left\{f_{A}\right\}_{A \in \mathcal{A}}$. Hence the collection of distinct implicit operations on $\mathcal{C}$ has a cardinality.

We improve this result as follows.

Theorem 10.2. For a finitary operational signature $\Sigma$, every full subcategory $\mathcal{S}$ of $\operatorname{Str} \Sigma$ definable with sufficiently low complexity has only a set of implicit operations.

Proof. As shown in the proof of Theorem 7.5, for each object $Y$ of $\mathcal{S}$ the slice category $(\mathcal{S} \cap H(\kappa) \downarrow Y)$ is cofinal in $(\mathcal{K} \downarrow Y)$ for some regular cardinal $\kappa$, where $\mathcal{K}$ is the (essentially small) class of $\kappa$-presentable objects in $\operatorname{Str} \Sigma$. Thus each object of $\mathcal{S}$ is a $\kappa$-filtered colimit of objects from the set $\mathcal{S} \cap H(\kappa)$. The rest is the same as in the proof of Theorem 10.1. 
Acknowledgments. We are much indebted to the referee for a deep and careful reading of the manuscript and a number of pertinent corrections. We also thank George Bergman for helpful remarks on the last section. The authors were supported by the Spanish Ministry of Science and Innovation under grants MTM2007-63277, MTM2008-03389, MTM2010-15831 and MTM201125229, by the Generalitat de Catalunya under grants 2005 SGR 606, 2005 SGR 738, 2009 SGR 119 and 2009 SGR 187, and by the Ministry of Education of the Czech Republic under project MSM0021622409. This research was supported through the Research in Pairs programme by the Mathematisches Forschungsinstitut Oberwolfach in 2008.

\section{References}

[1] Adámek, J., Herrlich, H., Strecker, G.: Abstract and Concrete Categories. Wiley, New York (1990); reprinted in Repr. Theory Appl. Categ. 17 (2006) Zbl 1113.18001 MR 2240597

[2] Adámek, J., Rosický, J.: Locally Presentable and Accessible Categories. London Math. Soc. Lecture Note Ser. 189, Cambridge Univ. Press, Cambridge (1994) Zbl 0795.18007 MR 1294136

[3] Adámek, J., Rosický, J.: On preaccessible categories. J. Pure Appl. Algebra 105, 225-232 (1995) Zbl 0852.18003 MR 1367868

[4] Adams, J. F.: A variant of E. H. Brown's representability theorem. Topology 10, 185-198 (1971) Zbl 0197.19604 MR 0283788

[5] Adams, J. F.: Localisation and Completion, with an Addendum on the Use of BrownPeterson Homology in Stable Homotopy. Lecture notes by Z. Fiedorowicz on a course given at the University of Chicago in Spring 1973; revised and supplemented by Z. Fiedorowicz. arXiv:1012.5020 (2010) MR 0420607

[6] Almeida, J.: Finite Semigroups and Universal Algebra. World Sci. (1994) Zbl 0844.20039 MR 1331143

[7] Bagaria, J.: $C^{(n)}$ cardinals. Arch. Math. Logic 51, 213-240 (2012) Zbl 1250.03108 MR 2899689

[8] Bagaria, J., Brooke-Taylor, A.: On colimits and elementary embeddings. J. Symbolic Logic 78, 562-578 (2013) Zbl 1275.03144 MR 3145196

[9] Bagaria, J., Casacuberta, C., Mathias, A. R. D.: Epireflections and supercompact cardinals. J. Pure Appl. Algebra 213, 1208-1215 (2009) Zbl 1160.03034 MR 2497569

[10] Bergman, G. M.: An Invitation to General Algebra and Universal Constructions. 2nd ed., Universitext, Springer, New York (2015); 1st ed., Henry Helson, Berkeley (1998) Zbl 0917.00001 MR 1650275

[11] Bousfield, A. K.: The localization of spaces with respect to homology. Topology 14, 133-150 (1975) Zbl 0309.55013 MR 0380779

[12] Bousfield, A. K.: Constructions of factorization systems in categories. J. Pure Appl. Algebra 9, 207-220 (1976/77) Zbl 0361.18001 MR 0478159

[13] Bousfield, A. K., Friedlander, E. M.: Homotopy theory of $\Gamma$-spaces, spectra, and bisimplicial sets. In: Geometric Applications of Homotopy Theory, Lecture Notes in Math. 658, Springer, Berlin, 80-130 (1978) Zbl 0405.55021 MR 0513569

[14] Brooke-Taylor, A.: Indestructibility of Vopěnka's Principle. Arch. Math. Logic 50, 515-529 (2011) Zbl 1222.03051 MR 2805294

[15] Casacuberta, C., Chorny, B.: The orthogonal subcategory problem in homotopy theory. In: An Alpine Anthology of Homotopy Theory, Contemp. Math. 399, Amer. Math. Soc., Providence, 41-53 (2006) Zbl 1106.55005 MR 2222503

[16] Casacuberta, C., Gutiérrez, J. J., Rosický, J.: Are all localizing subcategories of stable homotopy categories coreflective? Adv. Math. 252, 158-184 (2014) Zbl 06284319 MR 3144227 
[17] Casacuberta, C., Scevenels, D., Smith, J. H.: Implications of large-cardinal principles in homotopical localization. Adv. Math. 197, 120-139 (2005) Zbl 1082.55007 MR 2166179

[18] Chorny, B.: Localization with respect to a class of maps I-Equivariant localization of diagrams of spaces. Israel J. Math. 147, 93-139 (2005) Zbl 1276.55024 MR 2166357

[19] Chorny, B.: Abstract cellularization as a cellularization with respect to a set of objects. In: Categories in Algebra, Geometry and Mathematical Physics, Contemp. Math. 431, Amer. Math. Soc., Providence, 165-170 (2007) Zbl 1134.55014 MR 2342827

[20] Dror Farjoun, E.: Homotopy localization and $v_{1}$-periodic spaces. In: Algebraic Topology: Homotopy and Group Cohomology, Lecture Notes in Math. 1509, Springer, Berlin, 104-113 (1992) Zbl 0808.55008 MR 1185964

[21] Dror Farjoun, E.: Cellular Spaces, Null Spaces and Homotopy Localization. Lecture Notes in Math. 1622, Springer, Berlin (1996) Zbl 0842.55001 MR 1392221

[22] Eda, K.: A Boolean power and a direct product of abelian groups. Tsukuba J. Math. 6, 187193 (1982) Zbl 0533.20026 MR 0705112

[23] Eklof, P. C., Mekler, A. H.: Almost Free Modules: Set-Theoretic Methods. North-Holland, Amsterdam (1990); revised ed.: North-Holland Math. Library 65, North-Holland, Amsterdam (2002) Zbl 1054.20037 MR 1055083(I) MR 1914985(II)

[24] Freyd, P. J.: Homotopy is not concrete. In: The Steenrod Algebra and its Applications, Lecture Notes in Math. 168, Springer, Berlin, 25-34 (1970); reprinted in Repr. Theory Appl. Categ. 6, 1-10 (2004) Zbl 1057.18001 MR 2118307

[25] Freyd, P. J., Kelly, G. M.: Categories of continuous functors I. J. Pure Appl. Algebra 2, 169191 (1972) Zbl 0257.18005 MR 0322004

[26] Gabriel, P., Ulmer, F.: Lokal präsentierbare Kategorien. Lecture Notes in Math. 221, Springer, Berlin (1971) Zbl 0225.18004 MR 0327863

[27] Goerss, P. G., Jardine, J. F.: Simplicial Homotopy Theory. Progr. Math. 174, Birkhäuser, Basel (1999); 2nd printing: Modern Birkhäuser Classics (2009) Zbl 0949.55001 MR 1711612

[28] Hirschhorn, P. S.: Model Categories and Their Localizations. Math. Surveys Monogr. 99, Amer. Math. Soc., Providence (2003) Zbl 1017.55001 MR 1944041

[29] Hovey, M.: Cohomological Bousfield classes. J. Pure Appl. Algebra 103, 45-59 (1995) Zbl 0841.55008 MR 1354066

[30] Jech, T.: Set Theory. Pure Appl. Math., Academic Press, New York (1978) Zbl 0419.03028 MR 0506523

[31] Jech, T.: Set Theory: The Third Millenium Edition, Revised and Expanded. Springer Monogr. Math., Springer, Berlin (2003) Zbl 1007.03002 MR 1940513

[32] Kan, D. M.: On c.s.s. complexes. Amer. J. Math. 79, 449-476 (1957) Zbl 0078.36901 MR 0090047

[33] Kanamori, A.: The Higher Infinite: Large Cardinals in Set Theory from Their Beginnings. Perspectives in Math. Logic, Springer, Berlin (1994) Z Zbl 0813.03034 MR 1321144

[34] Kunen, K.: Elementary embeddings and infinitary combinatorics. J. Symbolic Logic 36, 407 413 (1971) MR 0311478

[35] Kunen, K.: Set Theory: An Introduction to Independence Proofs. Stud. Logic Found. Math. 102, North-Holland, Amsterdam (1980) Zbl 0443.03021 MR 0597342

[36] Mac Lane, S.: Categories for the Working Mathematician. 2nd ed., Grad. Texts in Math. 5, Springer, New York (1998) Zbl 0906.18001 MR 1712872

[37] Magidor, M.: On the role of supercompact and extendible cardinals in logic. Israel J. Math. 10, 147-157 (1971) Zbl 0263.02034 MR 0295904

[38] Makkai, M., Paré, R.: Accessible Categories: The Foundations of Categorical Model Theory. Contemp. Math. 104, Amer. Math. Soc., Providence (1989) Zbl 0703.03042 MR 1031717 
[39] Mathias, A. R. D.: Weak systems of Gandy, Jensen and Devlin. In: Set Theory (Barcelona, 2003-2004), Trends Math., Birkhäuser, Basel, 149-224 (2006) Zbl 1113.03046 MR 2267149

[40] May, J. P.: Simplicial Objects in Algebraic Topology. Van Nostrand Math. Stud. 11, Van Nostrand (1967) Zbl 0165.26004 MR 0222892

[41] Nešetřil, J.: A rigid graph for every set. J. Graph Theory 39, 108-110 (2002) Zbl 1002.05068 MR 1880889

[42] Przeździecki, A. J.: An "almost" full embedding of the category of graphs into the category of groups. Adv. Math. 225, 1893-1913 (2010) Zbl 1217.18005 MR 2680194

[43] Rosický, J., Tholen, W.: Left-determined model categories and universal homotopy theories. Trans. Amer. Math. Soc. 355, 3611-3623 (2003) Z Zbl 1030.55015 MR 1990164

[44] Rudyak, Yu. B.: On Thom Spectra, Orientability, and Cobordism. Springer Monogr. Math., Springer, Berlin (1998) Zbl 0906.55001 MR 1627486

[45] Trnková, V., Adámek, J., Rosický, J.: Topological reflections revisited. Proc. Amer. Math. Soc. 108, 605-612 (1990) Zbl 0694.18006 MR 0987614 